\title{
Spatial renewable resource extraction under possible regime shift*
}

\author{
Christopher Costello ${ }^{\dagger} \quad$ Bruno Nkuiya $^{\ddagger} \quad$ Nicolas Quérou $^{\S}$
}

\begin{abstract}
How will countries harvesting mobile natural resources react to the possibility of regime shift? We address the non-cooperative exploitation of a migratory renewable resource in the presence of possible regime shift that affects its movement. Motivated by the anticipated effects of climate change, we model a regime shift that will alter the spatial movement patterns of the resource at some point in the future. We develop a stochastic spatial bioeconomic model to address the effects of this class of regime shift on non-cooperative harvest decisions made by decentralized owners such as countries exploiting a migratory fish or other natural resource stock. We find that the threat of a future shift modifies the standard golden rule and may induce more aggressive harvest everywhere, irrespective of whether the owner will be advantaged or disadvantaged by the shift. We also identify conditions under which the threat of regime shift induces owners to reduce harvest rates in advance of the shift. Our analysis suggests that different property rights structures (single ownership vs common property) or heterogeneous growth can give rise to previously unexplored incentives and can even reverse conventional wisdom about how countries will react to the prospect of future environmental change.
\end{abstract}

Keywords: Regime shift; spatial management; renewable resources; property rights

JEL Classification Codes: C73; H23; H73; Q22

${ }^{*}$ We are grateful for useful comments from seminar participants at various institutions including University of Alberta and EAERE 2016. Christopher Costello acknowledges the Sustainable Fisheries Group and Environmental Market Solutions Lab at UC Santa Barbara for helpful comments and financial support. Bruno Nkuiya gratefully acknowledges financial support by the Social Sciences and Humanities Research Council of Canada (SSHRC). Nicolas Quérou acknowledges financial support by the ANR project GREEN-Econ (Grant ANR-16-CE03-0005).

${ }^{\dagger}$ Bren School, UC Santa Barbara; and NBER. E-mail:costello@bren.ucsb.edu.

${ }_{\ddagger}^{\ddagger}$ University of Alberta, Edmonton, AB, T6C 4G9, Canada. E-mail:nkuiyamb@ualberta.ca.

${ }^{\S}$ CEE-M, Univ. Montpellier, CNRS, INRA, SupAgro, Montpellier, France. E-mail:nicolas.querou@supagro.fr. 


\section{Introduction}

Because renewable resources such as fish, water, game, and infectious diseases are mobile, extraction and productivity in one location affect economic opportunities in other locations. The resulting spatial externality can be dealt with by a central planner using a number of instruments including spatial taxes, limits of extractive effort, or other means. But in practice, many spatially connected renewable resources are managed via private property rights where autonomous entities (such as countries, villages, cooperatives or individual property right owners) choose their own extraction rates, taking as given the mobility of the resource and the extraction of their competitors. Indeed spatial property rights are implicitly the default approach for managing many renewable resources, ${ }^{1}$ despite their potential for inducing spatial externalities driven by resource mobility. This general problem has become a canonical model in spatial resource economics. The main finding from that literature is that non-cooperative extraction will necessarily entail over-extraction (relative to a social optimum) because no single owner is incentivized to account for the effects of her extraction on others (Kaffine and Costello 2011; Fenichel et al. 2014). The aggregate effects of this non-cooperation can range from extremely deleterious (see White and Costello (2014)) to practically insignificant (see Gisser and Sanchez (1980)). ${ }^{2}$

This growing literature has evolved in a deterministic setting in which productivity and dispersal functions are common knowledge and fixed over time. Rather, a growing scientific literature suggests that global change may induce regime shifts that affect resource dynamics, and thus may alter future economic incentives and returns. While there are many types of documented (and speculated) regime shifts, they generally share three common features. First, regime shifts tend to be abrupt - over a relatively short period of time they can shift the state of the world from one state to another. Second, the occurrence date of a regime shift is probabilistic - while scientists might have a sense of the likelihood of a regime shift 
occurring, we do not know with certainty when it will occur. Third, many regime shifts are thought to be irreversible - once the shift occurs, due to hysteresis, it may be difficult to return to its pre-shift state (Scheffer et al. 2001). ${ }^{3}$

Facing this type of regime shift fundamentally alters the constraints and incentives faced by property owners who extract mobile renewable resources. To our knowledge, the earliest contribution along these lines is McKelvey et al. (2003), who use numerical simulations to examine cooperative and non-cooperative behavior in a "fish war" game. Our approach is to tackle this challenge theoretically in a dynamic game, and to obtain analytical results. We are motivated by regime shifts such as those that may be driven by climate change, which could affect the spatial range or dispersal of a migratory fish species such as tuna. For example, under pre-shift parameters, suppose tuna tend to migrate equally between countries $A$ and $B$. But if a regime shift were to occur, the migratory pattern may shift to favor country $A$. If that kind of shift were predictable, it would clearly alter the incentives of countries $A$ and $B$ : Recognizing the improvement in future conditions, country $A$ might be willing to forego harvest today to build resource stocks and capitalize on improved future conditions. ${ }^{4}$ And recognizing the deterioration of future conditions, country $B$ would, intuitively, increase its current extraction. These intuitive predictions of countries' behaviors are consistent with results in Hannesson (2007) and Diekert and Nieminen (2015), but they turn out to be vulnerable to strategic interactions across players. This stylized example illustrates our key inquiry: How will the presence of a possible future regime shift alter strategic interactions of private property owners who extract a mobile natural resource? Will the threat of regime shift always entail a loss of precaution by one agent and an increase in precaution by the other? We address these and related questions in our investigation.

This paper builds on an emerging literature on natural resource management that addresses related questions, but in a context where the random occurrence of a regime shift inflicts a permanent loss to all harvesters. Polasky et al. (2011) and Ren and Polasky (2014) focus 
on optimal management whereas Fesselmeyer and Santugini (2013), Sakamoto (2014), Miller and Nkuiya (2016), and Diekert (2017) analyze the strategic management of a common pool resource. These contributions consider only scenarios in which all harvesters are identical and do not explicitly take into account the spatial movement of the resource. But since we are primarily interested in strategic interactions, our analysis investigates cases in which regime shift will alter the distribution of resource stocks so as to create winners and losers. ${ }^{5}$ We thus explicitly take into account the spatial movement of the resource and consider heterogenous harvesters subject to different, but connected, economic, environmental, and biological conditions. In this expanded setting we are able to derive tractable, analytical results, and dissect how features such as heterogeneity and movement change incentives and outcomes relative to the previous literature.

Our analysis thus contributes to an increasingly relevant class of economic papers addressing renewable resource management under the prospect of regime shift in the resource distribution, which advantages one patch and disadvantages the other patch. To gain traction on this challenging problem, existing papers rely either on numerical simulations alone, or require assumptions that we will relax. For example, we will allow for common property in each patch and for biological growth conditions to differ across patches. ${ }^{6}$ Prominent previous contributions include Diekert and Nieminen (2015) and Liu and Heino (2013) that concentrate on deterministic regime shift processes with finite transitional periods, and find that the harvester gaining the stock conserves it, whereas the one losing the stock acts aggressively. Hannesson (2007) considers a random regime shift process and exogenously assumes that escapement is constant within each environmental regime. He finds that the stock cannot go extinct whenever the intrinsic growth parameter is not too low (and smaller than one) and the shares of the stock are sufficiently heterogenous. And while McKelvey et al. (2003) allow for a fairly general stochastic model, they restrict analysis to numerical simulations. In this paper, we consider analytically the strategic interactions across agents and adopt 
a feedback approach such that in a given period, escapement endogenously depends on environmental conditions and the resource stock. The setting we analyze builds on, and attempts to generalize, several of the prominent early papers in this area; we argue that these generalizations deliver insights about new harvest incentives that enhance our understanding of strategic interactions over a spatial resource subject to regime shift.

The simplest case is when marginal harvesting costs are constant. In that setting, analytical findings reveal that harvesters in the advantaged patch (the ones gaining the stock) conserve the resource stock in the initial period whereas those in the disadvantaged patch (those losing the stock) increase initial harvest in response to the possible future shift in the resource distribution. In the steady state, we identify two opposing mechanisms through which uncertainty about the shift affects harvest decisions. The first is a direct effect of the shift that tends to reduce harvest incentives in the disadvantaged patch (relative to the no-shift case). The second mechanism is the strategic effect that tends to enhance harvest in the disadvantaged patch, because harvesters in the advantaged patch conserve the resource stock in the short run, which has a positive effect on the disadvantaged patch in the long run through migration. Interestingly, we find that each of these mechanisms can dominate the other depending on environmental, biological, and economic conditions.

To better understand the effects of uncertainty about the shift, we also investigate the case in which harvesting costs decline as the resource stock increases. We find that a stock effect plays a pivotal role in extraction incentives. When the stock effect is sufficiently strong, we find that the conventional wisdom is weakened or even reversed.

The paper unfolds as follows. Section 2 presents the model. Section 3 focuses on behavior following the regime shift. Section 4 analyses harvesters' incentives in anticipation of a future regime shift. Section 5 extends the analysis to the case where each patch is itself common property. Section 6 provides an illustrative example and some further discussion of corner solutions. Section 7 concludes. 


\section{The model}

A renewable resource stock is distributed heterogeneously across an ecosystem consisting of two areas or "patches," $A$ and $B$. Patches may differ in shape, size, environmental, and economic characteristics; for example, patches may be countries, private lands, or communal harvesting areas. The time index is denoted by $t=1,2,3, \ldots$ and $h_{j t}$ represents the extraction in patch $j$ during period $t$. The resource stock at the beginning of period $t$ in a given patch $j$ is denoted by $x_{j t}$ while the remaining residual stock (or "escapement") $e_{j t}$ is defined as $e_{j t} \equiv x_{j t}-h_{j t}$, which is the post-harvest stock at the end of period $t$. As such, when there is no harvest, say, in patch $j$, the current escapement is equal to the current resource stock: $e_{j t}=x_{j t}$.

Resource mobility will induce a spatial connection across patches. In period $t$, a fraction $K_{i j t}$ of patch $i$ 's resource stock moves to patch $j, i \neq j$ while the fraction $K_{i i t}$ stays within patch $i$. Therefore, $K_{i j t}+K_{i i t} \leq 1$ for $i, j \in\{A, B\}$ with $i \neq j$. In the case where this inequality is not binding, a fraction of the resource population living in patch $i$ moves out of the system at date $t$. The current resource distribution across patches is determined by the $2 \times 2$ dispersal matrix $K_{t}$, whose element $K_{i j t}$ takes the value $D_{i j}$ before the shift and $D_{i j}^{s}$ after the shift.

[Figure 1 Here]

At the beginning of the initial period, dispersal is in its pre-shift form, so $K_{i j 0}=D_{i j}$. Regime shift occurs at an unknown future date denoted by $\tau>0$ (that may be infinite), and dispersal irreversibly shifts to regime $s$, characterized by a dispersal matrix with terms $D_{i j}^{s}$. We assume that the shift will give a bio-physical advantage to region $A$ and a disadvantage to region $B$, so $D_{B A}^{s}>D_{B A} \geq 0, D_{B B}>D_{B B}^{s} \geq 0, D_{A B}>D_{A B}^{s} \geq 0$, and $D_{A A}^{s}>D_{A A} \geq 0$. Figure 1 illustrates the pre-shift (top panel) and post-shift (bottom panel) migration patterns where the arrow thickness and size of the patches indicate the strength of connectivity. 
Dispersal is thus characterized as follows:

$$
K_{i j t}=\left\{\begin{array}{lll}
D_{i j} & \text { for } & t<\tau, \\
D_{i j}^{s} & \text { for } \quad t \geq \tau \quad \text { for } \quad i, j=A, B .
\end{array}\right.
$$

The regime shift process described above can be represented by the stochastic process $\ell_{t}$ that may either take the values $I$ (for "initial") or $S$ (for "shift") with transition probabilities

$$
\begin{aligned}
& P\left(\ell_{t+1}=S \mid \ell_{t}=S\right)=1 ; \quad P\left(\ell_{t+1}=S \mid \ell_{t}=I\right)=\lambda, \\
& P\left(\ell_{t+1}=I \mid \ell_{t}=I\right)=1-\lambda .
\end{aligned}
$$

At the outset of the initial period, the resource stocks $x_{A 0}$ and $x_{B 0}$ in patches $A$ and $B$ are known. In the absence of harvest, the resource stock grows according to the growth and dispersal equation

$$
x_{j t+1}=\sum_{i=A, B} g_{i}\left(x_{i t}\right) K_{i j t}, \quad j=A, B,
$$

where $g_{i}($.$) represents patch i$ 's growth function that satisfies standard conditions. It is increasing, concave and twice continuously differentiable.

In the presence of harvest, growth depends on escapement, so the law of motion becomes

$$
x_{j t+1}=\sum_{i=A, B} g_{i}\left(e_{i t}\right) K_{i j t}, \quad j=A, B .
$$

The evolution of the resource population is stochastically determined by harvest, growth, and environmental conditions $K_{i j t}$. The timing is thus: the present period stock $\left(x_{j t}\right)$ is observed and then harvested $\left(h_{j t}\right)$ giving escapement $\left(e_{j t}\right)$, which then grows $\left(g_{j}\left(e_{j t}\right)\right)$, and disperses to itself $\left(K_{j j t}\right)$ and to the other patch $\left(K_{j i t}\right)$.

Suppose now that each patch is owned by a single entity. For example, this could be a Territorial User Right Fishery (TURF), a farm on which bees reside, or a country hosting 
migratory fish, game, or waterfowl. We allow for, but do not require, prices and costs to be patch specific. Denote by $p_{j}$ the unit price associated with patch $j$; costs may depend on the stock size and are treated later in this section.

The harvester's period- $t$ harvest is $\left(x_{j t}-e_{j t}\right)$ so, in the absence of harvesting costs, her period- $t$ profit is $p_{j}\left(x_{j t}-e_{j t}\right)$. In the presence of harvesting costs, the period- $t$ profit for harvester $j$ is simply the integral of profit flows over the entire extractive period and can be written as follows

$$
\pi_{j t}=\int_{e_{j t}}^{x_{j t}}\left[p_{j}-c_{j}(v)\right] d v .
$$

Here, the term $c_{j}(v)$ is the marginal harvesting cost when the available stock is $v$. The integral $\int_{e_{j t}}^{x_{j t}} c_{j}(v) d v$ in Equation 5 represents the total cost of harvest during period-t, which may be patch specific. We assume that $c_{j}^{\prime}(v) \leq 0$, that is, the marginal cost weakly decreases in the resource stock level. The rationale is that at a given date, a larger resource stock entails a smaller marginal harvest cost. In the case where $c_{j}^{\prime}()=$.0 , the profit in patch $j$ is linear in harvest; profit is strictly concave in harvest as long as $c_{j}^{\prime}()<$.0 (i.e. a stock effect is present). To determine whether or not the marginal cost is constant is an empirical issue (see for instance, Atewamba and Nkuiya (2017), for the case of non-renewable resources). We will show that a sufficiently strong stock effect can fundamentally alter harvest incentives, particularly in the presence of regime shift. We separately examine both cases below.

In our model, regime shift affects the geographic distribution of the resource stock, but does not inherently affect $p_{j}$ or $c_{j}(v)$. In reality, shifts in prices and costs may also occur for example as range shifts change the location of harvest (which could affect fishing costs) or as the supply chain is altered (which could affect prices). As long as after the regime shift, these changes still adhere to our main assumptions (marginal fishing cost is a function of contemporaneous stock and price is constant in a period and exogenous to harvest), then these kinds of shifts in economic parameters could be accommodated in our modeling 
framework. But for more complicated kinds of economic shifts (for example, if the regime shift induces a downward-sloping demand curve), a new model would be required, which is unlikely to have analytically tractable results. For all of these reasons, we focus on the case when prices are constant (though may be patch specific), marginal harvest costs depend only on current resource stock in each patch, and regime shift affects only the geographic distribution of the stock.

Prior to the shift, i.e. for any date $t<\tau$, the present value payoff function in patch $j=A, B$ is given by

$$
\sum_{k=t}^{\tau-1} \delta^{(k-t)}\left[p_{j}\left(x_{j k}-e_{j k}\right)-\int_{e_{j k}}^{x_{j k}} c_{j}(v) d v\right]+\delta^{\tau-t} W_{j}(x(\tau)),
$$

where $W_{j}(x(\tau))$ represents the period- $\tau$ continuation value of the problem for that harvester and $\delta$ is the discount factor. We next solve the post-shift problem and use its result to derive the complete solution for the uncertain regime shift problem presented above.

\section{The post-shift problem}

In this section we examine the game between harvesters that will occur following the regime shift. We follow the growing literature, starting with the seminal paper of Reed (1979), that uses escapement as the control variable. ${ }^{7}$ In this setting, the patch- $j$ harvester chooses an escapement strategy to maximize her present discounted profits taking as given the escapement strategy of her rival. Thus, immediately following the regime shift, the owner of patch $j$ solves:

$$
\begin{aligned}
& W_{j}\left(x_{\tau}\right)=\max _{e_{j t}, t \geq \tau} \sum_{k=\tau}^{+\infty} \delta^{(k-\tau)}\left[p_{j}\left(x_{j k}-e_{j k}\right)-\int_{e_{j k}}^{x_{j k}} c_{j}(v) d v\right], \\
& \text { subject to (4) with } x_{\tau} \equiv\left(x_{A \tau}, x_{B \tau}\right) \text { given. }
\end{aligned}
$$


We seek a Markov Perfect Nash Equilibrium (MPNE), which will define the equilibrium harvest decisions $\left(e_{A}\left(x_{A}, x_{B}\right), e_{B}\left(x_{A}, x_{B}\right)\right)$ following the regime shift. The escapement decision rule $\left(e_{A}\left(x_{A}, x_{B}\right), e_{B}\left(x_{A}, x_{B}\right)\right)$ is a MPNE if, given the resource stock at the outset of period $\tau\left(x_{\tau} \equiv\left(x_{A \tau}, x_{B \tau}\right)\right)$, at any date $t \geq \tau,\left\{e_{j}\left(x_{A s}, x_{B s}\right), s \geq t\right\}$ is a solution to the optimization problem above. The feedback Nash equilibrium is a MPNE and can be found by specifying and manipulating the Bellman equations for both players. The Bellman equation for the harvester operating in patch $j$ can be written as:

$$
W_{j}\left(x_{t}\right)=\max _{e_{j t}}\left\{p_{j}\left(x_{j t}-e_{j t}\right)-\int_{e_{j t}}^{x_{j t}} c_{j}(v) d v+\delta W_{j}\left(x_{t+1}\right)\right\},
$$

which is subject to (4) with the initial resource stock $x_{\tau} \equiv\left(x_{A \tau}, x_{B \tau}\right)$ given.

The first-order conditions require

$$
p_{j}=c_{j}\left(e_{j t}\right)+\delta \sum_{i=A, B} \frac{\partial W_{j}}{\partial x_{i t+1}}\left(x_{t+1}\right) g_{j}^{\prime}\left(e_{j t}\right) D_{j i}^{s}, \quad j=A, B .
$$

This equation states that patch $j$ chooses her escapement level to equate the resource price with its augmented marginal cost, which is the marginal cost, augmented by the value forgone by harvesting today rather than keeping the resource for future harvests. The challenge is that the form of the value function $W_{j}(x)$ is unknown. However, its properties can be derived given the structure of this problem. These derivations allow us to characterize an equilibrium over the post regime shift phase, summarized as follows:

Lemma 1. Over the post regime shift phase, the following results hold.

(i) Patch $j$ is harvested down to the escapement level $e_{j}$. These state-independent (i.e. constant) escapements constitute an equilibrium of the game and are given by the solution to 


$$
p_{j}-c_{j}\left(e_{j}\right)=\delta D_{j j}^{s}\left[p_{j}-c_{j}\left(g_{j}\left(e_{j}\right) D_{j j}^{s}+g_{i}\left(e_{i}\right) D_{i j}^{s}\right)\right] g_{j}^{\prime}\left(e_{j}\right), \quad i=A, B \quad \text { and } \quad i \neq j
$$

(ii) Each patch's equilibrium resource stock reaches its steady state in one period, and is thereafter time-independent.

Proof. All proofs reside in the appendix.

Equation 7 implicitly defines harvester $j$ 's best response function, $e_{j}\left(e_{i}\right)$, and suggests that her actions depend on the flow of the resource to her own patch $\left(D_{i j}^{s}\right)$, but that $e_{j}\left(e_{i}\right)$ does not depend on $D_{j i}^{s}$ nor $D_{i i}^{s}, i \neq j$, which are terms defining the resource flow to the other patch. This is the case because harvester $j$ does not ascribe any value to additional resource stock located out of its boundaries (i.e. $\frac{\partial W_{j}}{\partial x_{i t}}\left(x_{t}\right)=0$ for $i \neq j$ ) because she knows that the best response of her rival would be to harvest any additional stock that arrives. The equilibrium escapement level corresponds to the intersection of best response functions $e_{A}\left(e_{B}\right)$ and $e_{B}\left(e_{A}\right)$. We can also employ this analysis to identify the equilibrium escapement level of the no-shift case, in which the resource distribution is deterministic and never shifts. We denote the no-shift variables by a tilde (e.g. $\left.\tilde{e}_{j t}\right)$. They can be retrieved from Condition 7 by replacing (for $i, j=A, B) D_{i j}^{s}$ by $D_{i j}$ and $\left(x_{A \tau}, x_{B \tau}\right)$ by $\left(x_{A 0}, x_{B 0}\right)$. Because the no-shift case takes the same form as the post shift case (albeit with different parameter values), the equilibrium escapement level of the no-shift case is also time and stock independent (see Lemma 1). The equilibrium resource stock outcome of the no-shift case converges to its steady state in the second period of the game.

To better understand the effects of the shift, we next compare the outcomes of the post regime shift case and the no-shift case.

Proposition 1. Assume that marginal costs are constant (i.e., $c_{j}^{\prime}(x)=0$ for all $x, j=A, B$ ). Over the post regime shift phase, the following results hold. 
(i) For $j=A, B, e_{j}$ is implicitly defined by:

$$
g_{j}^{\prime}\left(e_{j}\right)=\frac{1}{\delta D_{j j}^{s}} .
$$

(ii) Relative to the no-shift case, the equilibrium escapement level in the post regime shift problem is larger in patch $A$ and smaller in patch $B: e_{B t} \leq \tilde{e}_{B t}$ and $e_{A t} \geq \tilde{e}_{A t}$ for all $t \geq \tau$. (iii) At any date $t \geq \tau+1$, the equilibrium resource stock $\left(x_{j t}\right)$ in patch $j$ is greater relative to the no-shift case $\left(\tilde{x}_{j t}\right)$ if and only if $D_{i j}^{s}>\bar{D}_{j x}^{s}$.

(iv) At any date $t \geq \tau+1$, the equilibrium harvest rate $\left(h_{j t}\right)$ in patch $j$ is larger relative to the no-shift case $\left(\tilde{h}_{j t}\right)$ if and only if $D_{i j}^{s}>\bar{D}_{j h}^{s}$, where $\bar{D}_{j x}^{s}$ and $\bar{D}_{j h}^{s}$ depend only on $\delta, D_{j j}^{s}$, $D_{A A}, D_{B B}$ and $D_{i j}, i=A, B, i \neq j$, and are given in the appendix.

Result $(i)$ of Proposition 1 suggests that harvester $j=A, B$ chooses her escapement level to equate the biological return of the resource discounted by the patch retention rate $\left(D_{j j}^{s}\right)$ and the financial rate of return. This is a non-cooperative "golden rule" for spatial growth models (Kaffine and Costello 2011), where $D_{j j}^{s}$ acts like an additional discount factor. Result (ii) of Proposition 1 is driven by the facts that $(a)$ in each patch, the equilibrium escapement level and the patch retention rate are positively related; $(b)$ patch $B$ 's retention rate decreases with the shift whereas this result is reversed for patch $A$. Results (iii) and (iv) of Proposition 1 lead to an unexpected outcome. Despite the fact that the shift inflicts biophysical damages to patch $B$ (i.e., $D_{A B}^{s}<D_{A B}$ and $D_{B B}^{s}<D_{B B}$ ), the resource stock in patch $B$ may be larger depending on the resource growth and spatial characteristics. In addition, harvester $B$ may have incentives to increase her harvest compared to the no-shift case.

We have so far addressed the cases where the shift has already occurred and where the shift will never occur. We next use these results to completely characterize the equilibrium behavior prior to the shift. 


\section{Non-cooperative behavior in advance of a regime shift}

So far we have analyzed the deterministic spatial game induced either following an irreversible regime shift or in the complete absence of regime shift. But our central research question asks how harvesters interact under the threat of a possible regime shift in the future. In this section, we focus on harvesters' responses to the prospect of a future regime shift. Taking the escapement strategy of the other harvester as given, harvester $j=A, B$ chooses the escapement strategy that maximizes her expected present discounted net profits

$$
V_{j}\left(x_{t}\right)=\max _{e_{j s}, s \geq t} \mathbb{E} \sum_{k=t}^{+\infty} \delta^{(k-t)}\left[p_{j}\left(x_{j k}-e_{j k}\right)-\int_{e_{j k}}^{x_{j k}} c_{j}(v) d v\right]
$$

which is subject to (4). We are interested in identifying a MPNE that we next derive using the feedback Nash equilibrium approach. Harvester $j$ 's value function given in (9) satisfies:

$$
V_{j}\left(x_{t}\right)=\max _{e_{j t}}\left[p_{j}\left(x_{j t}-e_{j t}\right)-\int_{e_{j t}}^{x_{j t}} c_{j}(v) d v+\delta(1-\lambda) V_{j}\left(x_{t+1}\right)+\delta \lambda W_{j}\left(x_{t+1}^{s}\right)\right]
$$

subject to (4), where $x_{j t+1}^{s}=g_{j}\left(e_{j t}\right) D_{j j}^{s}+g_{i}\left(e_{i t}\right) D_{i j}^{s}$ and $x_{j t+1}=g_{j}\left(e_{j t}\right) D_{j j}+g_{i}\left(e_{i t}\right) D_{i j}$. The first two terms on the right hand side of Equation 10 are just contemporaneous revenue and cost from harvesting the resource in patch $j$. The third term is the discounted expected value in the case where the regime shift does not occur at the end of period $t$ (this occurs with probability $(1-\lambda))$. The final term is the discounted expected value in the case where regime shift does occur at the end of period $t$, in which case we invoke the value functions from the post regime shift problem derived in Section 3 (this occurs with probability $\lambda$ ). 
To interpret Equation 10, it is instructive to rewrite it as follows:

$$
V_{j}\left(x_{t}\right)=\max _{e_{j t}}\left[p_{j}\left(x_{j t}-e_{j t}\right)-\int_{e_{j t}}^{x_{j t}} c_{j}(v) d v+\tilde{\delta}_{j} V_{j}\left(x_{t+1}\right)\right]
$$

where $\tilde{\delta}_{j}=\delta+\delta \lambda\left[W_{j}\left(x_{t+1}^{s}\right)-V_{j}\left(x_{t+1}\right)\right] / V_{j}\left(x_{t+1}\right)$ can be thought of as a risk-adjusted discount factor. Equation 11 can be interpreted as the Bellman equation associated with a deterministic model in which the discount rate endogenously accounts for the possibility of regime shift.

The first-order condition for this maximization problem can be written as

$p_{j}=c_{j}\left(e_{j t}\right)+\delta \lambda g_{j}^{\prime}\left(e_{j t}\right) \sum_{i=A, B} \frac{\partial W_{j}}{\partial x_{i t+1}}\left(x_{t+1}^{s}\right) D_{j i}^{s}+\delta(1-\lambda) g_{j}^{\prime}\left(e_{j t}\right) \sum_{i=A, B} \frac{\partial V_{j}}{\partial x_{i t+1}}\left(x_{t+1}\right) D_{j i}, \quad j=A, B$.

Under the assumptions adopted above, since $x_{A t+1}, x_{B t+1}, x_{A t+1}^{s}$ and $x_{B t+1}^{s}$ depend on $e_{A t}$, $e_{B t}$ and do not explicitly depend on $x_{A t}$ and $x_{B t}$, this optimality condition suggests that $e_{A t}$ and $e_{B t}$ are time and stock independent. This intuition is verified in the following lemma.

Lemma 2. Prior to the spatial regime shift, the following results hold:

(i) The pair $\left(e_{A}, e_{B}\right)$ constitutes a MPNE, where $e_{j}$ is implicitly defined as follows:

$$
\begin{aligned}
p_{j}-c_{j}\left(e_{j}\right) & =\delta \lambda D_{j j}^{s}\left[p_{j}-c_{j}\left(g_{j}\left(e_{j}\right) D_{j j}^{s}+g_{i}\left(e_{i}\right) D_{i j}^{s}\right)\right] g_{j}^{\prime}\left(e_{j}\right) \\
& +\delta(1-\lambda) D_{j j}\left[p_{j}-c_{j}\left(g_{j}\left(e_{j}\right) D_{j j}+g_{i}\left(e_{i}\right) D_{i j}\right)\right] g_{j}^{\prime}\left(e_{j}\right), \quad i \neq j .
\end{aligned}
$$

(ii) $e_{j}$ is stock and time independent.

(iii) A given patch equilibrium resource stock is time dependent and reaches its steady state in the second period.

Lemma 2 suggests that the MPNE in escapement has a simple structure that depends on spatial characteristics, but is state independent. As such, the equilibrium escapement level in 
patch $j$ is simply $e_{j}$ as defined in (12). In contrast to results obtained in Lemma 1, Equation 12 suggests that the escapement level in a patch depends on the probability of regime shift, the patch's self retention rate before and after the shift. Moreover, in (12), terms multiplying $\lambda$ capture harvester $j$ 's strategic responses to the threat of regime shift. Interestingly, for the particular case where $\lambda=0,(12)$ characterizes the equilibrium escapement levels for the no-shift case. The outcome of Lemma 2 allows us to derive the following results.

Proposition 2. Assume that marginal costs are constant (i.e., $c_{j}^{\prime}(x)=0$ for all $x, j=A, B$ ). Over the pre-regime shift phase, the following results hold:

(i) The equilibrium escapement level in patch $j=A, B$, satisfies

$$
g_{j}^{\prime}\left(e_{j}\right)=\frac{1}{\delta\left(\lambda D_{j j}^{s}+(1-\lambda) D_{j j}\right)} .
$$

(ii) The equilibrium escapement level in $\left(\begin{array}{c}\text { Patch A } \\ \text { Patch B }\end{array}\right)$ is $\left(\begin{array}{c}\text { increasing } \\ \text { decreasing }\end{array}\right)$ in the likelihood of the shift:

$$
\frac{\partial e_{A}}{\partial \lambda}>0 \quad \text { and } \quad \frac{\partial e_{B}}{\partial \lambda}<0
$$

Result $(i)$ reveals that harvester $j$ chooses her escapement to equate the financial rate of return with the expected biological return, which is biological growth $g_{j}^{\prime}\left(e_{j}\right)$, discounted by patch $j$ 's expected retention rate, $\lambda D_{j j}^{s}+(1-\lambda) D_{j j}$. In other words, the non-cooperative golden rule (13) obtained in the certainty case is modified in response to the threat of future spatial regime shift. As the probability of the shift is raised, anticipating the shift, harvester $A$ adjusts her harvest decisions to increase escapement (to take advantage of improved future conditions), while harvester $B$ reduces her escapement in response to the threat (to extract the resource before it shifts migration out of her region). Since the no-shift outcome is the special case where $\lambda=0$, result ( $i i)$ of Proposition 2 implies that over the pre-regime shift phase, the equilibrium escapement level is lower in patch $B$ and larger in patch $A$, compared to the no-shift levels. 
These results contribute to the literature using escapement strategies as control variable to address the management of a renewable resource. The seminal paper by Reed (1979) established the optimality of constant escapement in a stochastic fishery model. Extending that model, for example, Costello et al. (2015) examine the implications of partial enclosure of a renewable common resource in a deterministic setting where the resource distribution regime never shifts; and Costello and Polasky (2008) focus on the effects of environmental variability on optimal spatial harvest responses. These papers find that the optimal escapement level is time and state independent (and are thus constant). The above results suggest that regime shift creates a discontinuity in the equilibrium escapement levels, so optimal escapements shift in response to the regime shift. This is consistent with previous analyses of optimal resource management of a single, aspatial stock under cyclical population dynamics (Carson et al. 2009) or with environmental predictions (Costello et al. 2001; Kennedy and Barbier 2013).

We have focused on the implications of spatial regime shift on escapement decisions. But we can also analyze the effects of regime shift on harvest and resource stock. We summarize these results as follows:

Proposition 3. Assume that marginal costs are constant (i.e., $c_{j}^{\prime}(x)=0$ for all $x, j=A, B$ ). Over the phase prior to the shift, the following results hold.

(i) $h_{B 0}>\tilde{h}_{B 0}$ and $h_{A 0}<\tilde{h}_{A 0}$.

(ii) At any date $t \geq 1, x_{B t}>\tilde{x}_{B t}$ if and only if $D_{A B}>\bar{D}_{B}^{x}$ and $x_{A t}>\tilde{x}_{A t}$ if and only if $D_{B A}<\bar{D}_{A}^{x}$.

(iii) At any date $t \geq 1, h_{B t}>\tilde{h}_{B t}$ if and only if $D_{A B}>\bar{D}_{B}^{h}$ and $h_{A t}>\tilde{h}_{A t}$ if and only if $D_{B A}<\bar{D}_{A}^{h}$, where $\bar{D}_{j}^{x}$ and $\bar{D}_{j}^{h}$ depend only on $\lambda, \delta, D_{k k}, D_{k k}^{s}, k=A, B$ and are given in the Appendix.

At the initial date, anticipating that a shift may occur in the future, harvester $B$ is more aggressive (harvests more than she would in the no-shift case) while harvester $A$ adopts 
precautionary behavior (reduces her harvest compared to the no-shift case), see Proposition 3i. This seems intuitive because harvester $B$ stands to lose from the regime shift. In the steady state, however, these strategic interactions may be altered to induce a larger or a smaller harvest rate in each patch depending on the values of the spatial characteristics.

To better understand the intuition underpinning this result, it is instructive to decompose the difference between the steady-state harvest rate for harvester $B$ under the threat and no-threat cases as follows:

$$
h_{B t}-\tilde{h}_{B t}=\underbrace{\left[g_{A}\left(e_{A}\right)-g_{A}\left(\tilde{e}_{A}\right)\right] D_{A B}}_{\text {Term } 1>0}+\underbrace{\left[\left(D_{B B} g_{B}\left(e_{B}\right)-e_{B}\right)-\left(D_{B B} g_{B}\left(\tilde{e}_{B}\right)-\tilde{e}_{B}\right)\right]}_{\text {Term } 2<0} .
$$

This condition shows that the effect of the threat of regime shift on harvester $B$ 's steady state harvest is driven by two opposite mechanisms, captured by the two underbraced terms in the right hand side. Harvester $B$ will tend to harvest less in the steady state because she harvests aggressively in the initial period. This effect (captured by Term 2) represents the direct effect of the threat and is negative. ${ }^{8}$ Moreover, harvests in both patches are linked: As harvester $A$ reduces her initial harvest, a larger stock will end up in patch $B$. Term 1 represents the strategic effect on resource growth; it is positive by Proposition 2 and the fact that function $g_{A}$ is increasing. This effect tends to raise harvester B's steady-state harvest rate under the threat.

As $D_{A B}$ is reduced, it is entirely possible that harvester $B$ decreases her own steady state harvest, and that harvester $A$ increases her harvest. Result (iii) of Proposition 3 provides conditions on $D_{A B}$ and $D_{B A}$ under which these statements hold.

For ease of exposition, we have primarily focused on the linear cost case. While this is a common assumption in resource economics, and delivers intuitive results, it fails to capture the stock effect under which the harvest cost increases as the resource stock tends to decline. Such a stock effect is present whenever $c_{j}^{\prime}(x)<0$. It turns out that the presence of a stock 
effect can fundamentally alter strategic behavior across patches. We find here that these altered strategic incentives can be sufficiently strong to reverse the conventional wisdom about how harvesters will react to the threat of regime shift. The results are summarized in the following proposition.

Proposition 4. In the case where the cost functions are non-linear, it is possible that $e_{A}<\tilde{e}_{A}$ and $e_{B}>\tilde{e}_{B}$.

This result suggests that despite the fact that the typical harvester in $B$ will be disadvantaged by the regime shift, she may increase escapement in the pre-shift phase. ${ }^{9}$ More precisely, the escapement level in patch $j$ under the threat is larger than under the no-threat case if and only if

$$
\begin{aligned}
\lambda D_{j j}^{s}\left[p_{j}-c_{j}\left(x_{j}^{s}\right)\right] g_{j}^{\prime}\left(e_{j}\right) & +(1-\lambda) D_{j j}\left[p_{j}-c_{j}\left(x_{j}\right)\right] g_{j}^{\prime}\left(e_{j}\right) \\
& >D_{j j}\left[p_{j}-c_{j}\left(\tilde{x}_{j}\right)\right] g_{j}^{\prime}\left(\tilde{e}_{j}\right)
\end{aligned}
$$

where $x_{j}^{s}=g_{j}\left(e_{j}\right) D_{j j}^{s}+g_{i}\left(e_{i}\right) D_{i j}^{s}, x_{j}=g_{j}\left(e_{j}\right) D_{j j}+g_{i}\left(e_{i}\right) D_{i j}$, and $\tilde{x}_{j}=g_{j}\left(\tilde{e}_{j}\right) D_{j j}+g_{i}\left(\tilde{e}_{i}\right) D_{i j}$.

Like the result in Proposition 2, this counterintuitive finding arises as a consequence of strategic interactions, except that here, strategic interactions are being driven by the stock effect. It is illustrative to consider the case of two countries harvesting a mobile fish stock. When the stock effect is absent (so $c^{\prime}=0$ ), there is no strategic feedback across countries. In other words, while the countries' harvest choices are inextricably linked, the escapement choices are not; when $c^{\prime}=0$, the escapement choice of country $B$ has no impact on the escapement choice of country $A$. In that setting, a country that stands to lose its stock in the future is incentivized to leave fewer fish in the water and a country that stands to gain its stock is incentivized to leave more fish in the water.

But when a stock effect is present $\left(c^{\prime}<0\right)$, the optimal escapement level of country $B$ does depend on the escapement level in country $A$. When country $A$ leaves more fish in the 
water, some of them swim to country $B$, so the stock of fish in $B$ is larger. This lowers the cost of fishing, and it turns out that this always leads country $B$ to leave more fish in the water. So, under the threat of regime shift such as climate change, there are two important effects on country $B$. First, country $B$ knows it will be disadvantaged by climate change, so it tends to want to leave fewer fish in the water. But, because country $A$ tends to leave more fish in the water (for precisely the same reason), spatial movement means it is possible that the stock of fish in country $B$ will be larger, which tends to make country $B$ want to leave more fish in the water. Which effect dominates will depend on the relative magnitudes of the two effects. Thus, it is quite possible that the country that will be disadvantaged by climate change may leave more fish in the water than under the no-threat case.

\section{An extension to common property}

While patches act non-cooperatively over harvest, we have assumed that each individual patch is harvested by a single owner. But in some settings, it is more realistic for patch $j$ to be common property, and is itself harvested by several non-cooperative agents. For example, if patches are the exclusive economic zones of countries, then harvesters would be individual fishing vessels within those territorial waters. The incentives engendered by this common property feature of non-cooperative spatial patches have not been analyzed previously. In this section we explore the role of common property in determining harvest and conservation incentives in anticipation of a regime shift.

To capture the common property feature, we generalize the previous model such that patch $j$ is now harvested by $N_{j} \geq 1$ harvesters acting non-cooperatively in all periods. Harvesters are identical within each patch, but we maintain the assumption that incentives may differ across patches with respect to economic, environmental, and biological conditions.

As we assumed previously, we denote by $p_{j}$ and $c_{j}$ the unit price and marginal harvesting 
cost function associated with patch $j$. Each of the $N_{j}$ harvesters in patch $j$ have access to an equal fraction of the stock, $\frac{x_{j t}}{N_{j}}$. This available stock will be extracted by harvester $k$ down to escapement level $\xi_{j t}^{(k)}$. Since harvesters are identical within each patch, the harvester's period- $t$ harvest is $\left(\frac{x_{j t}}{N_{j}}-\xi_{j t}^{(k)}\right)$ so, in the absence of harvesting costs, her period- $t$ profit is $p_{j}\left(\frac{x_{j t}}{N_{j}}-\xi_{j t}^{(k)}\right)$. In the presence of harvesting costs, the period- $t$ profit for harvester $k=$ $1,2, \ldots, N_{j}$ associated with patch $j$ can thus be written as

$$
\pi_{j t}^{(k)}=\int_{\xi_{j t}^{(k)}}^{\frac{x_{j t}}{N_{j}}}\left[p_{j}-c_{j}(v)\right] d v
$$

Recall that we are interested in a Markov Perfect Nash Equilibrium (MPNE), which determines the equilibrium escapement decision rules for all harvesters as functions of $\left(x_{A}, x_{B}\right)$ : $\left(\xi_{A}^{(1)}\left(x_{A}, x_{B}\right), \xi_{A}^{(2)}\left(x_{A}, x_{B}\right), \ldots, \xi_{A}^{\left(N_{A}\right)}\left(x_{A}, x_{B}\right), \xi_{B}^{(1)}\left(x_{A}, x_{B}\right), \ldots, \xi_{B}^{\left(N_{B}\right)}\left(x_{A}, x_{B}\right)\right)$. Since we are in a two-patch setting and harvesters operating in a given patch are identical, from now on, we concentrate on symmetric equilibria within each patch: $\xi_{A}()=.\xi_{A}^{(k)}($.$) and \xi_{B}()=.\xi_{B}^{(\ell)}($. for all $k, \ell$. Therefore, it suffices to find a pair of escapement strategies $\left(e_{A}(),. e_{B}().\right)$ with $e_{A}()=.N_{A} \xi_{A}($.$) and e_{B}()=.N_{B} \xi_{B}($.$) .$

Lemma 3. Prior to the spatial regime shift, the following results hold:

The pair $\left(e_{A}, e_{B}\right)$ constitutes a MPNE, where $e_{j}$ is implicitly defined as follows:

$$
\begin{aligned}
p_{j}-c_{j}\left(\frac{e_{j}}{N_{j}}\right) & =\delta \lambda D_{j j}^{s}\left[p_{j}-c_{j}\left(g_{j}\left(e_{j}\right) \frac{D_{j j}^{s}}{N_{j}}+g_{i}\left(e_{i}\right) \frac{D_{i j}^{s}}{N_{j}}\right)\right] \frac{g_{j}^{\prime}\left(e_{j}\right)}{N_{j}} \\
& +\delta(1-\lambda) D_{j j}\left[p_{j}-c_{j}\left(g_{j}\left(e_{j}\right) \frac{D_{j j}}{N_{j}}+g_{i}\left(e_{i}\right) \frac{D_{i j}}{N_{j}}\right)\right] \frac{g_{j}^{\prime}\left(e_{j}\right)}{N_{j}}, \quad i \neq j .
\end{aligned}
$$

This proposition provides an interesting characterization of the equilibrium strategic behaviors. In particular, it reveals that the equilibrium escapement levels prior to the shift depend on the biological return, $N_{j}$, economic and environmental conditions as well as the probability of spatial regime shift. Because the common property case considered in this 
section generalizes the model from Section 2 to $N_{j}$ harvesters, a natural question is how the number of harvesters affects strategic responses to the possible occurrence of the shift. We formally address this and related questions below. For the sake of tractability, we restrict our attention to the standard logistic growth function case, which can be written as follows

$$
g_{j}\left(e_{j}\right)=e_{j}+r_{j} e_{j}\left(1-\frac{e_{j}}{K_{j}}\right) \quad \text { for } \quad j=A, B
$$

where $K_{j}$ represents the carrying capacity and $r_{j}$ the intrinsic growth rate for patch $j$.

We first examine the effects on harvest decisions prior to the shift.

Proposition 5. Adopting $g_{j}$ as defined in (18) and provided marginal harvesting costs are constant:

(i) Patch j's equilibrium escapement, $e_{j}$, is decreasing in $N_{j}$ and satisfies

$$
g_{j}^{\prime}\left(e_{j}\right)=\frac{N_{j}}{\delta\left(\lambda D_{j j}^{s}+(1-\lambda) D_{j j}\right)} .
$$

(ii) Assume that $N_{A}=N_{B}=N$. In comparison with the no-shift case, the threat of a shift increases (resp. reduces) the steady-state harvest rate in patch $A$ (resp. patch B) whenever the inequalities $\delta\left(1+r_{A}\right) D_{A A}>N>N_{A}^{s}>0$ (resp. $\left.\delta\left(1+r_{B}\right) D_{B B}^{s}>N>N_{B}^{s}>0\right)$ hold. (iii) Assume that $N_{A} \neq N_{B}$. Relative to the no-shift case, the threat of a shift increases (resp. lowers) the steady-state harvest rate in patch $A$ (resp. patch B), but only if $N_{A}$ (resp. $\left.N_{B}\right)$ satisfies $\delta\left(1+r_{A}\right) D_{A A}>N_{A}>\hat{N}_{A}\left(\right.$ resp. $\left.\delta\left(1+r_{B}\right) D_{B B}^{s}>N_{B}>\hat{N}_{B}\right)$, where $N_{j}^{s}$ and $\hat{N}_{j}>0(j=A, B)$ are real numbers that depend on spatial characteristics and are given in the appendix.

In the above results, conditions $\delta\left(1+r_{A}\right) D_{A A}>N_{A}$ and $\delta\left(1+r_{B}\right) D_{B B}^{s}>N_{B}$ respectively ensure that, in each period, resource stock in patch $A$ (resp. $B$ ) is not fully depleted. 
These results highlight the implications of having more than one harvester in a patch. For instance, in the case where $N_{A}=N_{B}=1$, Result (ii) of Proposition 5 implies that the steady-state harvest rate in patch $A$ (resp. patch $B$ ) is lower (resp. higher) as long as $\min \left(N_{A}^{s}, \delta\left(1+r_{A}\right) D_{A A}\right)>1\left(\right.$ resp. $\left.\min \left(N_{B}^{s}, \delta\left(1+r_{B}\right) D_{B B}\right)>1\right)$ holds. In the case where $N_{A}=N_{B}=N \geq 2$ this result does not hold for patch $A($ resp. patch $B)$ when $\delta\left(1+r_{A}\right) D_{A A}>$ $N>N_{A}^{s}>1\left(\right.$ resp. $\left.\delta\left(1+r_{B}\right) D_{B B}>N>N_{B}^{s}>1\right)$ are satisfied.

The above results also highlight the importance of group size asymmetry (i.e., $N_{A} \neq N_{B}$ ). For example, in the case where the number of harvesters across patches is identical (i.e., $N_{A}=N_{B}=N \geq 1$ ), we show in the appendix that the steady-state harvest rate in patch $A$ (resp. patch $B$ ) is lower (resp. greater) irrespective the number of harvesters when $N_{A}^{s}<0$ (resp. $N_{B}^{s}<0$ ) holds. Irrespective the sign of $N_{A}^{s}$, Result (iii) of Proposition 5 highlights, however, the non-validity of that finding for patch $A$ when $N_{A} \neq N_{B}$ and $\delta\left(1+r_{A}\right) D_{A A}>N_{A}>\hat{N}_{A}$ hold. Similarly, the finding fails to hold for patch $B$ as long as $N_{B} \neq N_{A}$ and $\delta\left(1+r_{B}\right) D_{B B}>N_{B}>\hat{N}_{B}$ are satisfied. These findings show the importance of group size asymmetry. Whether $N_{A}$ or $N_{B}$ is small or large, our analysis reveals harvest responses to the threat that are qualitatively different than those in models where the number of players in both patches is identical (i.e., standard models in which there is a single agent in each patch).

It is also possible to derive the implications of considering common property vs. sole ownership and heterogenous growth on conservation. Our findings are summarized in the following proposition.

Proposition 6. Provided that $c_{j}^{\prime}()=$.0 and $g_{j}($.$) is defined in (18):$

(i) Patch $j$ is fully depleted in each period of time, but only if $N_{j} \geq \delta\left(1+r_{j}\right)\left(\lambda D_{j j}^{s}+(1-\lambda) D_{j j}\right)$ is satisfied.

(ii) In the case where $r_{j} \leq 1$, patch $j$ is fully depleted at each period of time whenever $N_{j} \geq 2$.

(iii) Patch $j$ is not fully depleted when $N_{j}=1, \lambda D_{j j}^{s}+(1-\lambda) D_{j j}>1 / \delta\left(1+r_{j}\right)$ and $r_{j} \leq 1$. 
Our motivation to tackle these extended results is that the related literature has focused on the cases in which $(i)$ the growth functions are logistic and identical with the intrinsic growth rate smaller than one; $(i i)$ each patch is owned by a single harvester. Relaxing each of those assumptions yields novel equilibria. For example, Proposition 6 suggests that different property rights structures may shift equilibrium states between depletion and no depletion (in each time period). More precisely, patch $j$ is fully depleted in each time period when $r_{j} \leq 1, c_{j}^{\prime}()=$.0 , and $N_{j} \geq 2$. In this context, when that patch is owned by a single agent and its expected self-retention rate is large, result ( $\mathrm{iii}$ ) shows that strategic harvest decisions no longer lead to full depletion.

Moreover, we find that when patches have different growth functions, equilibria arise that cannot be captured by models with identical growth functions. For instance, when $N_{j}=1$ and $c_{j}^{\prime}=0$, depletion is the equilibrium outcome in both patches when $r_{A}=r_{B}$ and

$$
\left.\delta\left(1+r_{j}\right) \times \min \left(\lambda D_{A A}^{s}+(1-\lambda) D_{A A}\right), \lambda D_{B B}^{s}+(1-\lambda) D_{B B}\right)<1
$$

However, only patch $B$ is fully depleted when $r_{A} \neq r_{B}, N_{j}=1, c_{j}^{\prime}=0$ and the following conditions hold:

$$
\left.\delta\left(1+r_{A}\right) \times\left[\lambda D_{A A}^{s}+(1-\lambda) D_{A A}\right)\right]>1 \text { and } \delta\left(1+r_{B}\right) \times\left[\lambda D_{B B}^{s}+(1-\lambda) D_{B B}\right] \leq 1 .
$$

These findings illustrate potential biases that may arise when heterogeneities in property rights structure or biological return exist, but are not taken into account.

Simultaneously accounting for common property and a stock effect on harvesting costs quickly becomes analytically intractable. But one way to get traction in the case where $c_{j}^{\prime}<0$ is as follows. Let $c_{i}^{\prime}=0$ and other model parameters be such that the optimal 
aggregate residual stock level $e_{i}$ does not depend on $N_{j}$ and is characterized by

$$
g_{i}^{\prime}\left(e_{i}\right)=\frac{N_{i}}{\delta\left(\lambda D_{i i}^{s}+(1-\lambda) D_{i i}\right)}
$$

Assume that the other patch is such that $c_{j}^{\prime}<0$ and initial model parameters (in particular the size of the user group, denoted $N_{j}^{0}$ ) are such that the optimal aggregate residual stock level $e_{j}$ is characterized by Condition 17. Assume moreover that $p_{j}-c_{j}(0)>0$ and $g_{j}^{\prime}($.$) is$ bounded from above. Then we have:

Proposition 7. There exists $\tilde{N}_{j}>N_{j}^{0}$ such that the resource will be fully depleted in patch $j$ at each time period for any size of user group greater than or equal to $\tilde{N}_{j}$.

This result reinforces the general intuition from above: Adding common property to the regime shift game induces a depletion threat that could jeopardize the very existence of the renewable resource. Thus, common property and regime shift spatial interactions are, in some sense, substitutes for one another. In our model, either is sufficient on its own to induce severe resource depletion (or, depending on harvesting costs, possibly full depletion), but the two can also work in tandem, where both effects in combination can be a double whammy for a renewable resource subject to spatial interactions and regime shift.

\section{Further discussion}

In this section we provide a brief numerical illustration and some further analysis regarding corner solutions.

\subsection{Illustrative Example}

To visualize and briefly illustrate our theoretical findings, we develop a simple, stylized numerical example. We assume growth in patch $j$ is logistic (as given in Equation 18), and 
that harvesting cost is linear in harvest, so Propositions 1, 2, 3, 5, and 6 apply. Baseline parameters are given as follows: $r_{A}=r_{B}=1.8, K_{A}=K_{B}=100, \delta=.95, D_{A A}=$ $.85, D_{B B}=.85, D_{A B}=.1, D_{B A}=.1, \lambda=.05$, and $N=2$. Regime shift results in $D_{A A}^{s}=.9, D_{B B}^{s}=.8, D_{A B}^{s}=.05$, and $D_{B A}^{s}=.15$. Our approach is to solve for the pre-regime-shift MPNE escapement levels under these baseline parameters, and then to derive the comparative statics of escapement with respect to each of these parameters.

To begin, suppose no regime shift was to occur. In that case, the socially-optimal escapements would have been $e_{A}=e_{B}=47.0$ (just under half of the carrying capacity in each patch). Again, with no threat of regime shift, if patches $A$ and $B$ compete with each other, equilibrium escapements in the two areas are both substantially lower $\left(e_{A}=e_{B}=9.0\right.$, about an $81 \%$ reduction in escapement in each patch). This reduction in conservation arises from two factors: First, the non-cooperative nature of exploitation leads each owner to over-extract the fishery before her competitor can. The second, larger influence arises because $N>1$, which gives rise to a commons problem internal to each patch.

Allowing for the regime shift (which, for this parametrization has a very low probability of occurrence each period of just $\lambda=5 \%$ ) alters the harvest incentives of both parties. Prior to the shift, the MPNE of the regime shift game is given by $e_{A}=9.2$ and $e_{B}=8.8$. Consistent with Proposition 2, the patch that will be advantaged by regime shift (patch $A$ ) increases escapement and the patch that will be disadvantaged decreases escapement, relative to the case without regime shift. After the regime shift occurs, escapement diverges still further to $e_{A}=12.8$ and $e_{B}=4.7$. For illustrative purposes, suppose the regime shift occurs at time 10. Then the top panel of Figure 2 shows escapement in each patch (as a fraction of $\left.K_{i}\right)$ during the pre-regime-shift phase and the post-regime-shift phase. The bottom panel of Figure 2 shows the pre-regime-shift steady state harvest as a function of the regime shift risk, $\lambda$. Consistent with Proposition 3, the bottom panel of Figure 2 shows the case in which as the threat of regime shift increases, steady state harvest in patch $A$ increases and steady 
state harvest in patch $B$ decreases.

[Figure 2 Here]

Continuing with the regime shift scenario, we ask how sensitive escapements in each patch are to changes in the underlying parameters. To examine this question, we calculate elasticity of pre-regime-shift escapement in patch $A$ with respect to each parameter. For example, we ask: If the growth rate increases by $1 \%$, by what percentage will escapement in patch $A$ increase? And because these elasticities need not be constant, we examine how the escapement changes for larger and larger changes in each parameter.

The top panel of Figure 3 displays these comparative statics results for six parameters. Consistent with our theory, most parameters have a positive effect on escapement (indicated by an upward-sloping relationship between the parameter and the escapement); the only parameter examined that has a negative effect on escapement is the number of harvesters in each patch, $N$. The bottom panel of Figure 3 shows the point estimates of the elasticity of escapement with respect to each parameter. For this parameterization, the parameters with the largest elasticities are the discount factor $(\delta$, whose elasticity is +7.6$)$, the number of users in patch $A\left(N_{A}\right.$, whose elasticity is -7.5), ${ }^{10}$ and the growth rate of the biological population in patch $A\left(r_{A}\right.$, whose elasticity is +4.5$)$. Under this parameterization, changes in the regime shift likelihood had a relatively small effect on optimal escapement in patch $A$ (elasticity of $<1$, though still positive).

[Figure 3 Here]

\subsection{Feasibility of interior solutions}

At this stage we briefly discuss the feasibility of interior solutions. ${ }^{11}$ We focus on the case of constant marginal costs so we can adopt general function forms for growth. First, the

feasibility of a strictly interior MPNE is ensured when the following conditions are satisfied, 
for any $j=A, B$ :

$$
g_{j}^{\prime}(0)>\frac{1}{\min \left\{\delta D_{j j}, \delta D_{j j}^{s}\right\}}, \quad g_{j}^{\prime}\left(x_{j 0}\right)<\frac{1}{\max \left\{\delta D_{j j}, \delta D_{j j}^{s}\right\}}, \quad g_{j}^{\prime}\left(x_{j}\right)<\frac{1}{\delta D_{j j}^{s}}
$$

where $x_{j}=D_{j j}^{s} g_{j}\left(e_{j}\right)+D_{i j}^{s} g_{i}\left(e_{i}\right)(i \neq j)$ and $e_{j}(j=A, B)$ denotes the pre-shift MPNE outcome in patch $j$. Under these conditions, following the shift, a corner solution where any agent would harvest nothing during a given time period is infeasible. In other words, for any $j=A, B$, denoting $e_{j}^{P}$ agent $j$ 's post-shift interior equilibrium strategy ${ }^{12}$, the feasibility of interior MPNE requires that $D_{j j}^{s} g_{j}\left(e_{j}\right)+D_{i j}^{s} g_{i}\left(e_{i}\right)>e_{j}^{P}$ be satisfied in the time period where the shift occurs. ${ }^{13}$ Due to the concavity of $g_{B}($.$) and the fact that D_{B B}>D_{B B}^{s}$, this condition is satisfied for $j=B$ without further assumptions. Regarding agent $A$, this condition is satisfied as long as $D_{B A}^{S} g_{B}\left(e_{B}\right)$ is sufficiently large. ${ }^{14}$

These conclusions allow us to derive conditions under which corner solutions may emerge. Specifically, if the following conditions hold:

$$
g_{A}^{\prime}(0)>\frac{1}{\delta D_{A A}}, \frac{1}{\delta D_{B B}^{s}} \geq g_{B}^{\prime}(0)>\frac{1}{\delta\left[\lambda D_{B B}^{s}+(1-\lambda) D_{B B}\right]} ;
$$

and

$$
g_{A}^{\prime}\left(x_{A 0}\right)<\frac{1}{\delta D_{A A}^{s}}, \quad g_{A}^{\prime}\left(D_{A A}^{s} g_{A}\left(e_{A}\right)+D_{B A}^{s} g_{B}\left(e_{B}\right)\right)<\frac{1}{\delta D_{A A}^{s}}
$$

then an interior post-shift equilibrium outcome is not feasible, but a MPNE exists where the induced pre-shift outcome is interior, and where agent $B$ fully depletes the resource in her own patch at any post-shift time period. Note finally that additional results on corner solutions are provided in Propositions 6 and 7: They rely on a specific form of the growth functions, but they highlight the differences induced by different ownership structures. 


\section{Conclusion}

One of the most widely anticipated effects of global environmental change is the shift in the spatial distribution and migration of natural resource stocks. While effects will range from moderate to severe, and are occasionally predictable, in most cases, the occurrence of these shifts is uncertain. We have examined the effects of the threat of future spatial regime shift on strategic interactions between spatial property rights owners harvesting a mobile natural resource. Because our model allows for different economic returns, heterogenous growth, stock effects on costs, and spatial migration of the resource, we have been able to extract a number of novel results about how strategic behavior interacts with the threat of spatial regime shift. Our main contribution is to examine how the prospect of a spatial regime shift will affect non-cooperative incentives and equilibrium behavior across heterogeneous property owners. We considered as a baseline the no-shift case in which the resource distribution is deterministic and never shifts; this amounts to a non-cooperative spatial game in a deterministic environment. Then, introducing the possibility of a future regime shift, we examined the non-cooperative behavior of competing spatial property rights holders across a range of shift magnitudes. We modeled spatial regime shift as an abrupt change in the biophysical conditions that govern dispersal of the resource. The shift confers a clear advantage to one harvester and a clear disadvantage to the other. Our focus is on how the harvesters compete prior to the shift (but with common knowledge about the likelihood of the shift).

Our analysis allows for an arbitrary degree of regime shift. In the extreme, the shift could irreversibly drive the entire resource population out of one of the patches and into the other. In keeping with the literature, we call this the "complete shift" case, where $D_{B B}^{s}=D_{A B}^{s}=0$ and $D_{B A}^{s}=1$. Using methods similar to those in Propositions 3 and 4 , our analysis yields qualitatively similar results as for the partial regime shift case. 
Whatever its magnitude, the threat of regime shift always increases initial harvest in the disadvantaged patch when harvest costs are linear. But we also found that strategic interactions can induce that patch to harvest less in steady state under the threat of regime shift. Indeed, this finding can maintain even under the threat of complete shift. The equilibrium escapement level in a patch depends on the resource price, marginal harvesting costs, but only when a patch is common property. We find that strategic interactions can even lead to full depletion, provided the common property feature is sufficiently strong relative to the growth of the resource. In the case where harvest costs are non-linear, new strategic incentives arise; this effect can even reverse the conventional wisdom, so even a patch that will be advantaged by the regime shift may act more aggressively in advance of the shift.

Our results may shed some light on the economic literature examining renewable resource management under the threat of a doomsday event (see for instance, Polasky et al. 2011). When the probability of regime shift is exogenous and utility is linear in harvest, the wisdom so far is that aggressive behavior always prevails prior to the shift. In this paper, the probability of regime shift is exogenous and a harvester makes her harvest decisions under the threat of the shift. In contrast to the aforementioned literature, we find conditions under which such harvesters may be cautious in response to disadvantageous regime shifts.

These results also relate to an interesting emerging policy debate. Many resource stocks such as marine fish, waterfowl, and some economically-significant game species migrate across national or other jurisdictions. At the same time, these migratory patterns are expected to change as a consequence of future climate change (Molinos et al. 2016). The results in this paper help inform our understanding about the behavioral responses of countries or other jurisdictions in advance of shifts, and unveil some counterintuitive predictions arising from strategic interactions to capture the resource. While informative in their own right, these results could be leveraged to inform policy responses for managing transboundary resources subject to possible future regime shift. 


\section{Notes}

${ }^{1}$ For example, migratory waterfowl and fish are managed by the multiple countries whose boundaries they traverse, groundwater is managed by overlying landowners, game is often managed by private wildlife management areas or hunting clubs, and invasive species are controlled by adjacent landowners.

${ }^{2}$ See Stavins (2011) for empirical evidence on tragedies of the commons.

${ }^{3}$ Issues of uncertainty and irreversibility have been analyzed in the context of the timing of environmental policy adoption (see Ulph and Ulph (1997) for an early contribution). They also matter significantly for problems of voluntary contributions to global public goods (see Elsayyad and Morath (2016) for issues raised by technology transfers, or Nordhaus (2015) and Harstad (2016) for discussions on the design of a climate treaty).

${ }^{4}$ See Costello et al. (2001) and Carson et al. (2009) for aspatial models of resource management with environmental predictions.

${ }^{5}$ For example, climate change may irreversibly trigger local scarcity or extinction in the sub-polar regions and invasion in the arctic for many species of fish (Cheung et al. 2009).

${ }^{6}$ In reality, habitat, salinity, water currents, nutrients, oxygen concentration and extraction costs may vary across patches leading to heterogenous biological, economic, and environmental conditions.

${ }^{7}$ This is a benign assumption because harvest and escapement are linked by the identity $h_{t} \equiv x_{t}-e_{t}$. This approach has subsequently been adopted by numerous authors including Costello and Polasky (2008), Costello et al. (2015), Kapaun and Quaas (2013), and many papers cited therein.

${ }^{8}$ To formally see why Term 2 is negative, it can be shown by using (13) that $D_{B B} g_{B}^{\prime}\left(e_{B}\right)-1>0$. For the special case where $\lambda=0$, we thus have $D_{B B} g_{B}^{\prime}\left(\tilde{e}_{B}\right)-1>0$. Combining this result with the facts that $\tilde{e}_{B}>e_{B}$ and the function $D_{B B} g_{B}(x)-x$ is concave, we conclude that $D_{B B} g_{B}\left(e_{B}\right)-e_{B}<D_{B B} g_{B}\left(\tilde{e}_{B}\right)-\tilde{e}_{B}$.

${ }^{9}$ Using the growth functions defined in (18) along with the marginal costs $c_{j}(v)=\theta_{j} / v, j=A, B$ and the set of parameters $\delta=0.95, x_{A 0}=0.84, x_{B 0}=0.8, D_{A A}=0.7, D_{B B}=0.77, D_{A B}=0.26, D_{B A}=0.22$, $D_{A A}^{s}=0.82, D_{B B}^{s}=0.65, D_{A B}^{s}=0.14, D_{B A}^{s}=0.28, K_{A}=0.45, K_{B}=3, N_{A}=2=N_{B}, r_{A}=3.55$, $r_{B}=3.45$, we find numerically that $e_{A}$ and $e_{B}$ are both increasing in $\lambda$.

${ }^{10}$ While $N$ should technically be an integer, we calculate the elasticity of escapement with respect to $N$ by varying it a small amount, to maintain consistency with the approach taken with other parameters.

${ }^{11}$ Further technical details are available from the authors upon request.

${ }^{12}$ This residual stock level is characterized by $e_{j}^{P}=\left(g_{j}^{\prime}\right)^{-1}\left(\frac{1}{\delta D_{j j}^{s}}\right)$ as shown in the analysis. 
${ }^{13}$ Another required condition is that $D_{j j}^{s} g_{j}\left(e_{j}^{P}\right)+D_{i j}^{s} g_{i}\left(e_{i}^{P}\right)>e_{j}^{P}$ holds for any agent $j$. Yet, it is satisfied for $j=A, B$ without further assumptions due to the concavity of $g_{j}($.$) and the characterization of e_{j}^{P}$.

${ }^{14} \mathrm{~A}$ sufficient condition is that $D_{B A}^{s} g_{B}\left(e_{B}\right) \geq D_{A A}^{s}\left[g_{A}\left(e_{A}^{P}\right)-g_{A}\left(e_{A}\right)\right]$ be satisfied, which is more likely as the growth parameter becomes larger in patch $B$, or as $\lambda D_{B B}^{s}+(1-\lambda) D_{B B}$ becomes smaller. 
Figures

Before the shift
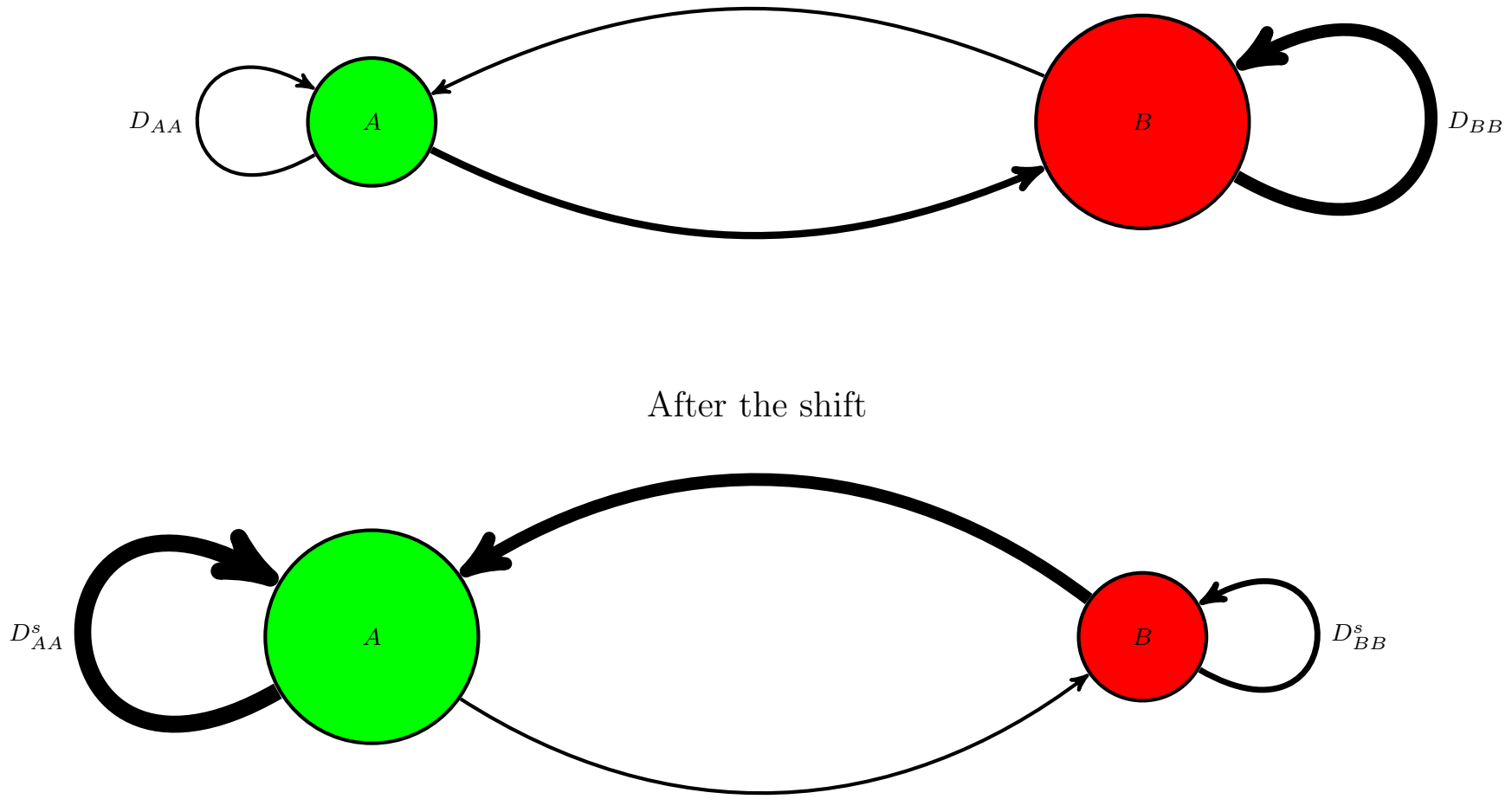

Figure 1 
Figure 2

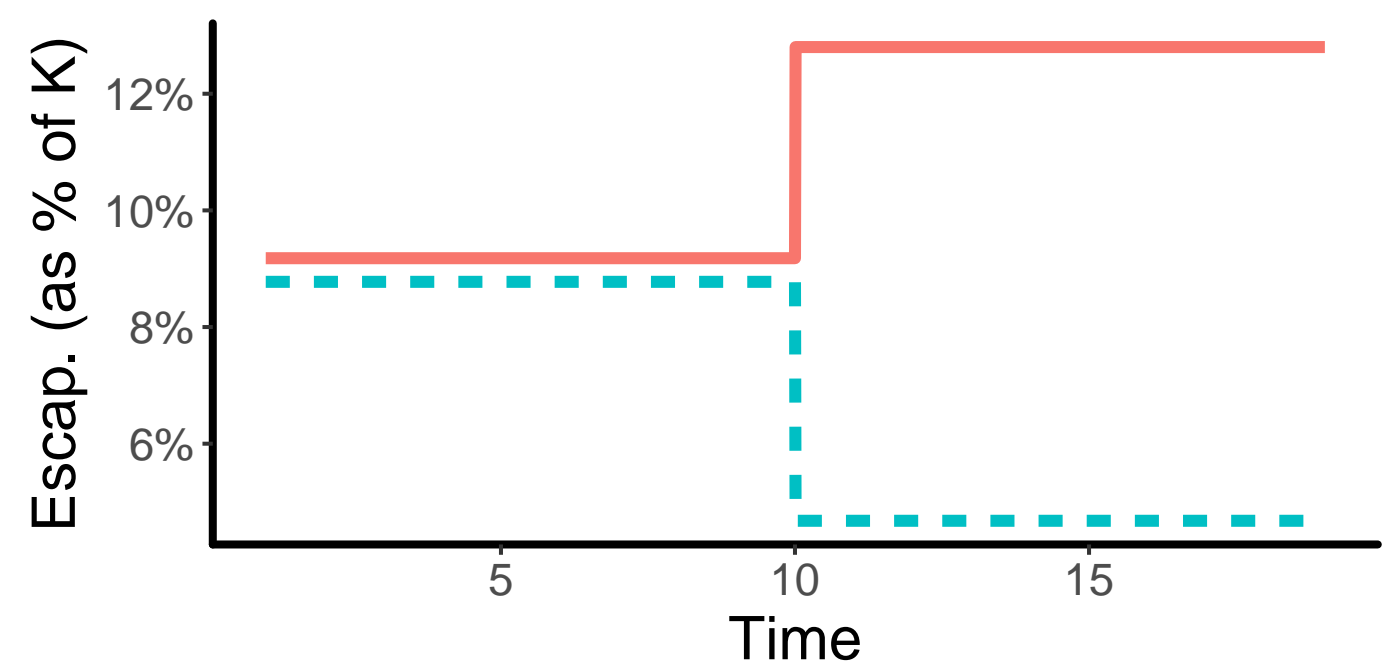

Patch

- A

- B

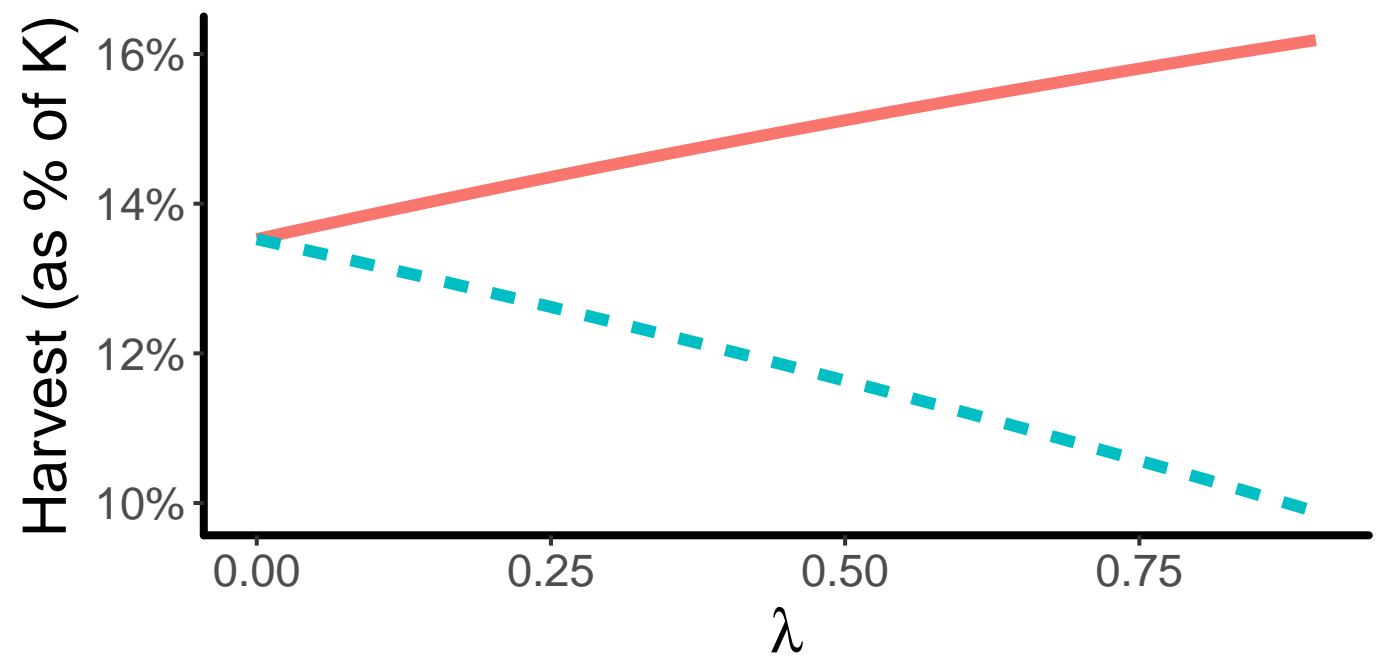

Patch

- A

- B 
Figure 3
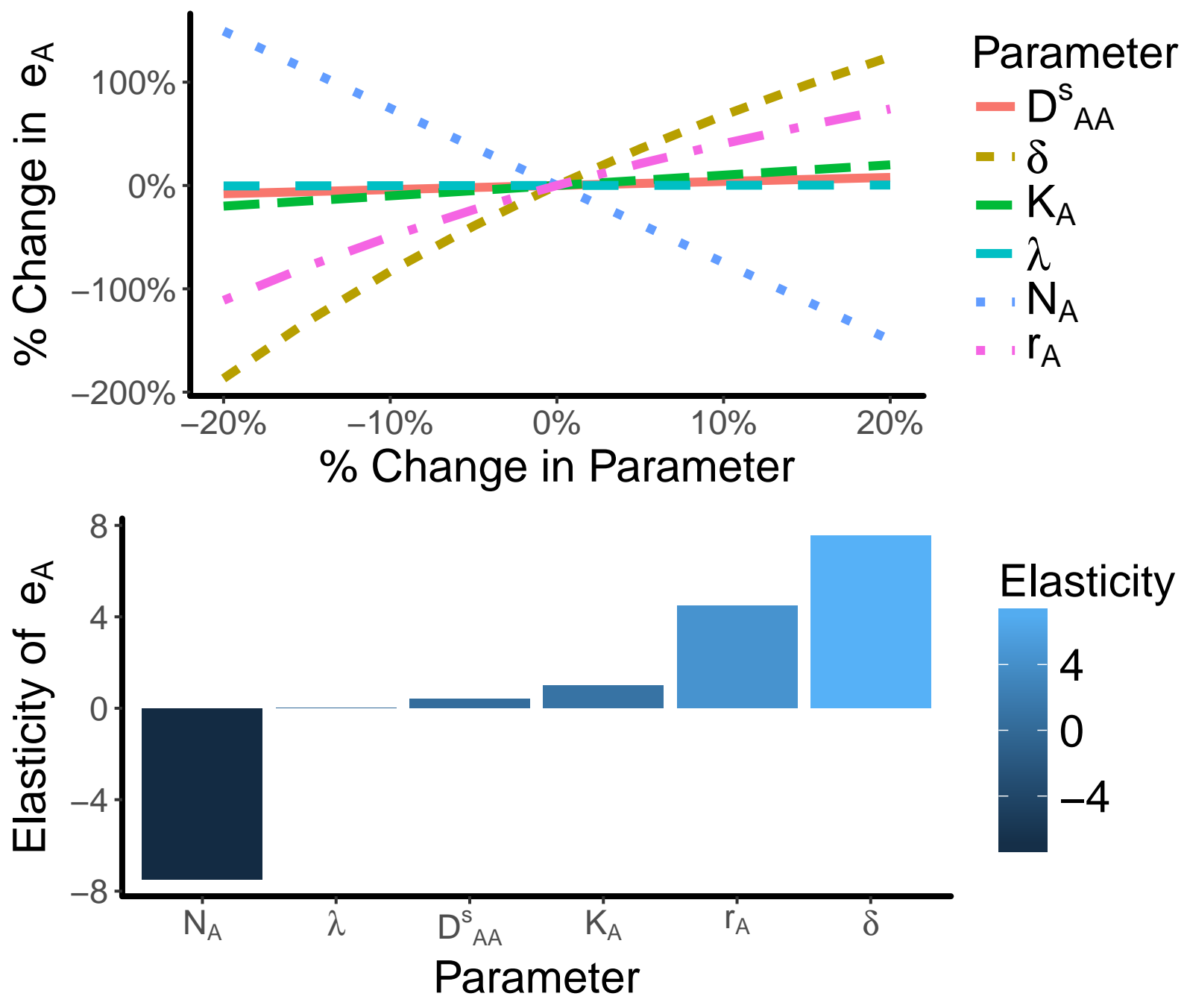


\section{Appendix}

\section{A Details for Section 3}

\section{Proof of Lemma 1}

(i) The equilibrium value function can be written as

$$
W_{j}\left(x_{t}\right)=p_{j}\left(x_{j t}-e_{j t}\right)-\int_{e_{j t}}^{x_{j t}} c_{j}(v) d v+\delta W_{j}\left(x_{t+1}\right)
$$

where the law of motion is:

$$
x_{j t+1}=\sum_{i=A, B} g_{i}\left(e_{i t}\right) K_{i j t}
$$

Note that the vector $x_{t+1}$ depends only on $e_{i}$ and $e_{j}$. The first-order condition is

$$
p_{j}=c_{j}\left(e_{j t}\right)+\delta \sum_{i=A, B} \frac{\partial W_{j}}{\partial x_{i t+1}}\left(x_{t+1}\right) g_{j}^{\prime}\left(e_{j t}\right) D_{j i}^{s}
$$

In general many possible equilibria can exist in this game. We show that a profile of state-independent strategies (where $e_{i}$ and $e_{j}$ are stock independent) solve the dynamic programming problem and therefore constitute an equilibrium of the model; we will focus on this class of solutions. Under these strategies, the first two terms in the first-order condition are independent of vector $x_{t}$ by inspection. The derivative of the value function in period $t+1$ depends on the period $t+1$ state, but is independent of the period $t$ state. For an interior solution we have $e_{i t}<x_{i t}$ and, using the expression of the law of motion, we conclude that $x_{i t+1}$ depends on $e_{i t}$ but not on $x_{i t}$. Therefore, the terms in the sum in the above first-order condition are independent of $x_{t}$. Under these assumptions, the period $t$ game problem has state independent strategies: $e_{i t}$ does not depend on $x_{t}$ for any $i=A, B$. Using the expression 
of the law of motion yields $\frac{\partial x_{j t+1}}{\partial e_{i t}}=D_{i j} g_{i}^{\prime}\left(e_{i t}\right)$ for any $j=A, B$, moreover we obtain

$$
\frac{\partial W_{j}}{\partial x_{i t}}\left(x_{t+1}\right)=0, \quad \text { for all } \quad i, j \in\{A, B\}, \quad i \neq j
$$

Combining this result along with (20), it follows that

$$
\frac{\partial W_{j}}{\partial x_{k t}}\left(x_{t}\right)= \begin{cases}p_{j}-c_{j}\left(x_{j t}\right) & \text { if } k=j \\ 0 & \text { if } k \neq j\end{cases}
$$

Substituting this expression and that of $\frac{\partial x_{j t+1}}{\partial e_{i t}}$ into the first-order condition we obtain the required condition. This necessary condition is also sufficient provided the assumption of concavity of returns in the strategy (residual stock level) is satisfied. Finally, since the growth function is independent of time, as is spread, self retention and economic costs, the optimal choice $e_{i t}$ is independent of time for any $i=A, B$.

(ii) This is a simple consequence of the fact that $e_{i}$ and $e_{j}$ are time and state independent.

\section{Proof of Proposition 1}

(i) In the case where $c_{j}^{\prime}(x)=0$ for all $x, j=A, B$, using the first-order condition

$$
p_{j}-c_{j}\left(e_{j}\right)=\delta D_{j j}^{s}\left[p_{j}-c_{j}\left(g_{j}\left(e_{j}\right) D_{j j}^{s}+g_{i}\left(e_{i}\right) D_{i j}^{s}\right)\right] g_{j}^{\prime}\left(e_{j}\right), \quad i=A, B \quad \text { and } \quad i \neq j .
$$

we obtain

$$
g_{j}^{\prime}\left(e_{j}\right)=\frac{1}{\delta D_{j j}^{s}}, \quad g_{j}^{\prime}\left(\tilde{e}_{j}\right)=\frac{1}{\delta D_{j j}} .
$$

(ii) Since $D_{A A}^{s}>D_{A A}$ and $D_{B B}>D_{B B}^{s}$, it follows that $g_{A}^{\prime}\left(e_{A}\right)<g_{A}^{\prime}\left(\tilde{e}_{A}\right)$ and $g_{B}^{\prime}\left(e_{B}\right)>$ $g_{B}^{\prime}\left(\tilde{e}_{B}\right)$. Hence, $\tilde{e}_{A}<e_{A}$ and $\tilde{e}_{B}>e_{B}$. This is the case because functions $g_{j}(),. j=A, B$ are concave such that functions $g_{j}^{\prime}(),. j=A, B$ are decreasing. 
(iii) For $t \geq \tau+1$ :

$x_{j t} \equiv g_{j}\left(e_{j}\right) D_{j j}^{s}+g_{i}\left(e_{i}\right) D_{i j}^{s}>g_{j}\left(\tilde{e}_{j}\right) D_{j j}+g_{i}\left(\tilde{e}_{i}\right) D_{i j} \equiv \tilde{x}_{j t}$ if and only if

$$
D_{i j}^{s}>\bar{D}_{j x}^{s} \equiv \frac{g_{j}\left(\tilde{e}_{j}\right) D_{j j}+g_{i}\left(\tilde{e}_{i}\right) D_{i j}-g_{j}\left(e_{j}\right) D_{j j}^{s}}{g_{i}\left(e_{i}\right)} .
$$

(iv) For $t \geq \tau+1$, the relation

$h_{j t} \equiv x_{j t}-e_{j t}=g_{j}\left(e_{j}\right) D_{j j}^{s}+g_{i}\left(e_{i}\right) D_{i j}^{s}-e_{j t}>g_{j}\left(\tilde{e}_{j}\right) D_{j j}+g_{i}\left(\tilde{e}_{i}\right) D_{i j}-\tilde{e}_{j t}=\tilde{x}_{j t}-\tilde{e}_{j t} \equiv \tilde{h}_{j t}$ holds if and only if

$$
D_{i j}^{s}>\bar{D}_{j h}^{s} \equiv \frac{g_{j}\left(\tilde{e}_{j}\right) D_{j j}+g_{i}\left(\tilde{e}_{i}\right) D_{i j}-g_{j}\left(e_{j}\right) D_{j j}^{s}+\left(e_{j}-\tilde{e}_{j}\right)}{g_{i}\left(e_{i}\right)} .
$$

Equation 21 suggests that for $j=A, B, e_{j}$ depends only on $\delta$ and $D_{j j}^{s}$ whereas for $j=A, B$,

$\tilde{e}_{j}$ depends only on $\delta$ and $D_{j j}$. As such, $\bar{D}_{j x}^{s}$ and $\bar{D}_{j h}^{s}$ depend only on $\delta, D_{j j}^{s}, D_{A A}, D_{B B}$, and $D_{i j}, i=A, B$ and $i \neq j$.

\section{Proof of Lemma 2}

Similar to the proof of Lemma 1.

\section{B Details for Section 4}

\section{Proof of Proposition 2}

(i) In the case where $c_{j}^{\prime}()=$.0 , the first order condition

$$
p_{j}-c_{j}\left(e_{j}\right)=\delta D_{j j}^{s}\left[p_{j}-c_{j}\left(g_{j}\left(e_{j}\right) D_{j j}^{s}+g_{i}\left(e_{i}\right) D_{i j}^{s}\right)\right] g_{j}^{\prime}\left(e_{j}\right), \quad i=A, B \text { and } i \neq j \text {. }
$$


simplifies to

$$
g_{j}^{\prime}\left(e_{j}\right)=\frac{1}{\delta\left(\lambda D_{j j}^{s}+(1-\lambda) D_{j j}\right)} .
$$

(ii) Using the implicit value theorem, $e_{j}$ is a continuously differentiable function of $\lambda$. We then differentiate both sides of (22) with respect to $\lambda$. Rearranging the outcome yields

$$
\frac{\partial e_{j}}{\partial \lambda}=\frac{D_{j j}-D_{j j}^{s}}{\left.g_{j}^{\prime \prime}\left(e_{j}\right)\left[\lambda D_{j j}^{s}+(1-\lambda) D_{j j}\right)\right]^{2}}, \quad \text { for } \quad j=A, B
$$

Since $g_{j}^{\prime \prime}\left(e_{j}\right)<0, D_{B B}>D_{B B}^{s}$ and $D_{A A}^{s}>D_{A A}$, the result follows.

\section{Proof of Proposition 3}

(i) Since $e_{A 0} \equiv x_{A 0}-h_{A 0}>\tilde{e}_{A} \equiv x_{A 0}-\tilde{h}_{A 0}$, we necessarily have $\tilde{h}_{A 0}>h_{A 0}$. Moreover, since $e_{B 0} \equiv x_{B 0}-h_{B 0}<\tilde{e}_{B} \equiv x_{B 0}-\tilde{h}_{B 0}$, we necessarily have $\tilde{h}_{B 0}<h_{B 0}$.

(ii) Using the facts that $x_{j t} \equiv g_{j}\left(e_{j}\right) D_{j j}+g_{i}\left(e_{i}\right) D_{i j}, \tilde{x}_{j t}=g_{j}\left(\tilde{e}_{j}\right) D_{j j}+g_{i}\left(\tilde{e}_{i}\right) D_{i j}, g_{A}\left(e_{A}\right)>$ $g_{A}\left(\tilde{e}_{A}\right)$, and $g_{B}\left(e_{B}\right)<g_{B}\left(\tilde{e}_{B}\right)$, we get $x_{B t}>\tilde{x}_{B t}$ if and only if

$$
D_{A B}>\bar{D}_{B}^{x} \equiv D_{B B} \frac{g_{B}\left(\tilde{e}_{B}\right)-g_{B}\left(e_{B}\right)}{g_{A}\left(e_{A}\right)-g_{A}\left(\tilde{e}_{A}\right)}
$$

Using a similar reasoning, we find that $x_{A t}>\tilde{x}_{A t}$ if and only if

$$
D_{B A}<\bar{D}_{A}^{x} \equiv D_{A A} \frac{g_{A}\left(e_{A}\right)-g_{A}\left(\tilde{e}_{A}\right)}{g_{B}\left(\tilde{e}_{B}\right)-g_{B}\left(e_{B}\right)}
$$

(iii) Using a similar method as for the proof of result (ii) along with the fact that $h_{j}=x_{j}-e_{j}, j=A, B$, we get

- $h_{B t}>\tilde{h}_{B t}$ if and only if

$$
D_{A B}>\bar{D}_{B}^{h} \equiv \frac{D_{B B}\left(g_{B}\left(\tilde{e}_{B}\right)-g_{B}\left(e_{B}\right)\right)-\left(\tilde{e}_{B}-e_{B}\right)}{g_{A}\left(e_{A}\right)-g_{A}\left(\tilde{e}_{A}\right)} .
$$


- $h_{A t}>\tilde{h}_{A t}$ if and only if

$$
D_{B A}<\bar{D}_{A}^{h} \equiv \frac{D_{A A}\left(g_{A}\left(e_{A}\right)-g_{A}\left(\tilde{e}_{A}\right)\right)-\left(e_{A}-\tilde{e}_{A}\right)}{g_{B}\left(\tilde{e}_{B}\right)-g_{B}\left(e_{B}\right)} .
$$

Notice that for $j=A, B, \bar{D}_{j}^{h}$ and $\bar{D}_{j}^{x}$ depend only on $\lambda, \delta, D_{k k}$, and $D_{k k}^{s}, k=A, B$.

\section{Proof of Proposition 4}

Recall that for $j=A, B, e_{j}$ satisfies the first-order condition $(j \neq i)$

$p_{j}-c_{j}\left(e_{j}\right)=\delta \lambda D_{j j}^{s}\left[p_{j}-c_{j}\left(g_{j}\left(e_{j}\right) D_{j j}^{s}+g_{i}\left(e_{i}\right) D_{i j}^{s}\right)\right] g_{j}^{\prime}\left(e_{j}\right)+\delta(1-\lambda) D_{j j}\left[p_{j}-c_{j}\left(g_{j}\left(e_{j}\right) D_{j j}+g_{i}\left(e_{i}\right) D_{i j}\right)\right] g_{j}^{\prime}\left(e_{j}\right)$

Hence, the left-hand side of condition

$$
\lambda D_{j j}^{s}\left[p_{j}-c_{j}\left(x_{j}^{s}\right)\right] g_{j}^{\prime}\left(e_{j}\right)+(1-\lambda) D_{j j}\left[p_{j}-c_{j}\left(x_{j}\right)\right] g_{j}^{\prime}\left(e_{j}\right)>D_{j j}\left[p_{j}-c_{j}\left(\tilde{x}_{j}\right)\right] g_{j}^{\prime}\left(\tilde{e}_{j}\right)
$$

is equal to $p_{j}-c_{j}\left(e_{j}\right)$ while the right-hand side corresponds to $p_{j}-c_{j}\left(\tilde{e}_{j}\right)$. So this condition can be rewritten $p_{j}-c_{j}\left(e_{j}\right)>p_{j}-c_{j}\left(\tilde{e}_{j}\right)$. Since function $\ell_{j}(x)=p_{j}-c_{j}(x)$ is increasing, we conclude that this condition holds if and only if $e_{j}>\tilde{e}_{j}$.

\section{Proof of Lemma 3}

Similar to the proof of Lemma 2. 


\section{Proof of Proposition 5}

(i) In the case where $c_{j}^{\prime}=0$, the first-order condition

$$
\begin{aligned}
p_{j}-c_{j}\left(\frac{e_{j}}{N_{j}}\right) & =\delta \lambda D_{j j}^{s}\left[p_{j}-c_{j}\left(g_{j}\left(e_{j}\right) \frac{D_{j j}^{s}}{N_{j}}+g_{i}\left(e_{i}\right) \frac{D_{i j}^{s}}{N_{j}}\right)\right] \frac{g_{j}^{\prime}\left(e_{j}\right)}{N_{j}} \\
& +\delta(1-\lambda) D_{j j}\left[p_{j}-c_{j}\left(g_{j}\left(e_{j}\right) \frac{D_{j j}}{N_{j}}+g_{i}\left(e_{i}\right) \frac{D_{i j}}{N_{j}}\right)\right] \frac{g_{j}^{\prime}\left(e_{j}\right)}{N_{j}}, \quad i \neq j
\end{aligned}
$$

simplifies to $g_{j}^{\prime}\left(e_{j}\right)=\frac{N_{j}}{\delta\left(\lambda D_{j j}^{s}+(1-\lambda) D_{j j}\right)}$. Using the fact that $g_{j}$ is concave and partially differentiating both sides of this equality and rearranging, it can be shown that $e_{j}$ is decreasing in $N_{j}$.

Moreover, using this first-order condition in the case of logistic growth functions, we derive

$$
e_{j}=\frac{K_{j}}{2 r_{j}}\left[1+r_{j}-\frac{N_{j}}{\delta\left(\lambda D_{j j}^{s}+(1-\lambda) D_{j j}\right)}\right]
$$

Notice that $e_{A}>0$ if and only if $\delta\left(1+r_{A}\right) D_{A A}>N_{A}$. Likewise, we have $e_{B}>0$, but only when $\delta\left(1+r_{B}\right) D_{B B}^{s}>N_{B}$.

(iii) Denote by $h_{A}^{s}$, the steady-state total harvest for patch $A$. According to the law of motion of the stock in patch $j$, we have $h_{A}^{s}=g_{A}\left(e_{A}\right) D_{A A}+g_{B}\left(e_{B}\right) D_{B A}-e_{A}$. Combining this result with (23) and keeping in mind that the growth function is logistic, we derive

$$
h_{A}^{s}-\left.h_{A}^{s}\right|_{\lambda=0}=\eta_{A}\left(\lambda D_{A A}^{s}+(2-\lambda) D_{A A}\right) N_{A}^{2}-2 \eta_{A} \delta\left(\lambda D_{A A}^{s}+(1-\lambda) D_{A A}\right) N_{A}-\xi_{A},
$$

where,

$\xi_{A}=\lambda D_{B A} K_{B} N_{B}^{2} \frac{\left(D_{B B}-D_{B B}^{s}\right)\left(\lambda D_{B B}^{s}+(2-\lambda) D_{B B}\right)}{r_{B} \delta^{2} D_{B B}^{2}\left(\lambda D_{B B}^{s}+(1-\lambda) D_{B B}\right)^{2}}>0 ; \eta_{A}=\frac{\lambda K_{A}\left(D_{A A}^{s}-D_{A A}\right)}{\delta^{2} D_{A A} r_{A}\left(\lambda D_{A A}^{s}+(1-\lambda) D_{A A}\right)^{2}}>0$.

Denote by $\hat{N}_{A}$ the unique positive root of (24). Clearly, $h_{A}^{s}>\left.h_{A}^{s}\right|_{\lambda=0}$ whenever $N_{A}>\hat{N}_{A}$.

(ii) A similar reasoning as for the proof of Result (ii) suggests that $\left.h_{B}^{s}\right|_{\lambda=0}>h_{B}^{s}$ whenever 
$N_{B}>\hat{N}_{B}$, where $\hat{N}_{B}$ is the unique positive root of the second degree polynomial:

$$
-\eta_{B}\left(\lambda D_{B B}^{s}+(2-\lambda) D_{B B}\right) N_{B}^{2}+2 \eta_{B} \delta\left(\lambda D_{B B}^{s}+(1-\lambda) D_{B B}\right) N_{B}+\xi_{B}=0,
$$

where,

$\xi_{B}=D_{A B} K_{A} N_{A}^{2} \frac{\left(D_{A A}^{s}-D_{A A}\right)\left(\lambda D_{A A}^{s}+(2-\lambda) D_{A A}\right)}{r_{A} \delta^{2} D_{A A}^{2}\left(\lambda D_{A A}^{s}+(1-\lambda) D_{A A}\right)^{2}}>0 ; \eta_{B}=\frac{K_{B}\left(D_{B B}-D_{B B}^{s}\right)}{\delta^{2} D_{B B} r_{B}\left(\lambda D_{B B}^{s}+(1-\lambda) D_{B B}\right)^{2}}>0$.

(ii) Assume that $N_{A}=N_{B}=N$. In this case, condition (24) simplifies to

$$
h_{A}^{s}-\left.h_{A}^{s}\right|_{\lambda=0}=\left[-\xi_{A}^{*}+\eta_{A}\left(\lambda D_{A A}^{s}+(2-\lambda) D_{A A}\right)\right] N^{2}-2 \eta_{A} \delta\left(\lambda D_{A A}^{s}+(1-\lambda) D_{A A}\right) N,
$$

where, $\xi_{A}^{*}=\xi_{A} / N^{2}$ does not depend on $N_{A}=N_{B}=N$. Hence, $h_{A}^{s}>\left.h_{A}^{s}\right|_{\lambda=0}$ if and only if $N>N_{A}^{s} \equiv \frac{\left.2 \eta_{A} \delta\left[\lambda D_{A A}^{s}+(1-\lambda) D_{A A}\right)\right]}{\eta_{A}\left(\lambda D_{A A}^{s}+(2-\lambda) D_{A A}\right)-\xi_{A}^{*}}$ and $N_{A}^{s}>0$. It is also important to notice that $h_{A}^{s}<\left.h_{A}^{s}\right|_{\lambda=0}$ irrespective of the number of harvesters whenever $N_{A}^{s}<0$.

A similar reasoning as for patch $A$ leads to: $h_{B}^{s}<\left.h_{B}^{s}\right|_{\lambda=0}$ if and only if $N>N_{B}^{s} \equiv$ $\frac{\left.2 \eta_{B} \delta\left[\lambda D_{B B}^{s}+(1-\lambda) D_{B B}\right)\right]}{\eta_{B}\left(\lambda D_{B B}^{s}+(2-\lambda) D_{B B}\right)-\xi_{B}^{*}}$ and $N_{B}^{s}>0$ where, $\xi_{B}^{*}=\xi_{B} / N^{2}$. Moreover, $h_{B}^{s}>\left.h_{B}^{s}\right|_{\lambda=0}$ irrespective of the number of harvesters whenever $N_{B}^{s}<0$.

\section{Proof of Proposition 6}

Using $g_{j}^{\prime}\left(e_{j}\right)=\frac{N_{j}}{\delta\left(\lambda D_{j j}^{s}+(1-\lambda) D_{j j}\right)}$ and keeping in mind that the growth function is logistic, it can be shown that if escapement for patch $j$ is strictly positive, it is necessarily given by (23).

(i) Using (23), we derive $e_{j} \leq 0$ iff $N_{j} \geq\left(1+r_{j}\right)\left(\lambda D_{j j}^{s}+(1-\lambda) D_{j j}\right)$. The result then follows.

(ii) Assume that $N_{j} \geq 2$ and $r_{j} \leq 1$. These assumptions imply $N_{j} \geq\left(1+r_{j}\right)\left(\lambda D_{j j}^{s}+\right.$ $\left.(1-\lambda) D_{j j}\right)$ such that by Result $(i)$, patch $j$ is harvested down to extinction. 
(iii) In the case where $N_{j}=1$, we can verify that $e_{j}$ defined in (23) is strictly positive as long as $r_{j} \leq 1$ and $\left.\left[\lambda D_{j j}^{s}+(1-\lambda) D_{j j}\right)\right]>1 / \delta\left(1+r_{j}\right)$. The result then follows.

\section{Proof of Proposition 7}

It suffices to show that $e_{j}$ cannot be positive for $N_{j}^{0}$ arbitrarily large. Using the first-order condition, if $e_{j}>0$ we know that the following inequality holds:

$$
\begin{aligned}
p_{j}-c_{j}\left(\frac{e_{j}}{N_{j}^{0}}\right) & =\delta \lambda D_{j j}^{s}\left[p_{j}-c_{j}\left(g_{j}\left(e_{j}\right) \frac{D_{j j}^{s}}{N_{j}^{0}}+g_{i}\left(e_{i}\right) \frac{D_{i j}^{s}}{N_{j}^{0}}\right)\right] \frac{g_{j}^{\prime}\left(e_{j}\right)}{N_{j}^{0}} \\
& +\delta(1-\lambda) D_{j j}\left[p_{j}-c_{j}\left(g_{j}\left(e_{j}\right) \frac{D_{j j}}{N_{j}^{0}}+g_{i}\left(e_{i}\right) \frac{D_{i j}}{N_{j}^{0}}\right)\right] \frac{g_{j}^{\prime}\left(e_{j}\right)}{N_{j}^{0}}, \quad i \neq j .
\end{aligned}
$$

Since $D_{i j}^{s} \frac{g_{i}\left(e_{i}\right)}{N_{j}^{0}} \rightarrow 0$ and $\frac{g_{j}^{\prime}\left(e_{j}\right)}{N_{j}^{0}} \rightarrow 0$ as $N_{j}^{0}$ gets arbitrarily large, the left hand side term of equation (25) goes to zero. However, its right hand side term remains positive (as $N_{j}^{0}$ gets arbitrarily large) because $p_{j}>c_{j}(0)$ and $c_{j}$ is decreasing. Therefore, as $N_{j}^{0}$ becomes arbitrary large, condition (25) (that characterizes interior solutions in escapement) cannot hold. Hence, there exists $\tilde{N}_{j}$ such that patch $j$ is fully depleted at each time period as long as $N_{j} \geq \tilde{N}_{j}$. 


\section{References}

Atewamba, C. and B. Nkuiya (2017). Testing the assumptions and predictions of the Hotelling model. Environmental and Resource Economics 66 (1), 169-203.

Carson, R. T., C. Granger, J. Jackson, and W. Schlenker (2009). Fisheries management under cyclical population dynamics. Environmental and Resource Economics 42(3), $379-410$.

Cheung, W. W., V. W. Lam, J. L. Sarmiento, K. Kearney, R. Watson, and D. Pauly (2009). Projecting global marine biodiversity impacts under climate change scenarios. Fish and Fisheries 10(3), 235-251.

Costello, C. and S. Polasky (2008). Optimal harvesting of stochastic spatial resources. Journal of Environmental Economics and Management 56(1), 1-18.

Costello, C., S. Polasky, and A. Solow (2001). Renewable resource management with environmental prediction. Canadian Journal of Economics/Revue canadienne d'économique 34(1), 196-211.

Costello, C., N. Quérou, and A. Tomini (2015). Partial enclosure of the commons. Journal of Public Economics 121, 69-78.

Diekert, F. K. (2017). Threatening thresholds? the effect of disastrous regime shifts on the non-cooperative use of environmental goods and services. Journal of Public Economics 147, 30-49.

Diekert, F. K. and E. Nieminen (2015). International fisheries agreements with a shifting stock. Dynamic Games and Applications, 1-27.

Elsayyad, M. and F. Morath (2016). Technology transfers for climate change. International Economic Review 57(3), 1057-1084.

Fenichel, E. P., T. J. Richards, and D. W. Shanafelt (2014). The control of invasive species 
on private property with neighbor-to-neighbor spillovers. Environmental and Resource Economics 59(2), 231-255.

Fesselmeyer, E. and M. Santugini (2013). Strategic exploitation of a common resource under environmental risk. Journal of Economic Dynamics and Control 37(1), 125-136.

Gisser, M. and D. A. Sanchez (1980). Competition versus optimal control in groundwater pumping. Water Resources Research 16(4), 638-642.

Hannesson, R. (2007). Global warming and fish migrations. Natural Resource Modeling 20(2), 301-319.

Harstad, B. (2016). The dynamics of climate agreements. Journal of the European Economic Association 14, 719-756.

Kaffine, D. T. and C. Costello (2011). Unitization of spatially connected renewable resources. The BE Journal of Economic Analysis 85 Policy 11(1).

Kapaun, U. and M. F. Quaas (2013). Does the optimal size of a fish stock increase with environmental uncertainties? Environmental and Resource Economics 54(2), 293-310.

Kennedy, C. J. and E. B. Barbier (2013). Renewable resource management with environmental prediction: the importance of structural specification. Canadian Journal of Economics/Revue canadienne d'économique 46(3), 1110-1122.

Liu, X. and M. Heino (2013). Comparing proactive and reactive management: managing a transboundary fish stock under changing environment. Natural Resource Modeling 26(4), 480-504.

McKelvey, R., K. Miller, and P. Golubtsov (2003). Fish-wars revisited: a stochastic incomplete-information harvesting game. Risk and Uncertainty in Environmental and Natural Resource Economics, 93-112.

Miller, S. and B. Nkuiya (2016). Coalition formation in fisheries with potential regime 
shift. Journal of Environmental Economics and Management 79, 189-207.

Molinos, J. G., B. S. Halpern, D. S. Schoeman, C. J. Brown, W. Kiessling, P. J. Moore, J. M. Pandolfi, E. S. Poloczanska, A. J. Richardson, and M. T. Burrows (2016). Climate velocity and the future global redistribution of marine biodiversity. Nature Climate Change 6(1), 83-88.

Nordhaus, W. (2015). Climate clubs: overcoming free-riding in international climate policy. American Economic Review 105(4), 1339-1370.

Polasky, S., A. de Zeeuw, and F. Wagener (2011). Optimal management with potential regime shifts. Journal of Environmental Economics And Management 62, 229-240.

Reed, W. J. (1979). Optimal escapement levels in stochastic and deterministic harvesting models. Journal of environmental economics and management 6(4), 350-363.

Ren, B. and S. Polasky (2014). The optimal management of renewable resources under the risk of potential regime shift. Journal of Economic Dynamics and Control 40, 195-212.

Sakamoto, H. (2014). Dynamic resource management under the risk of regime shifts. Journal of Environmental Economics and Management 68(1), 1-19.

Scheffer, M., S. Carpenter, J. A. Foley, C. Folke, and B. Walker (2001). Catastrophic shifts in ecosystems. Nature 413(6856), 591.

Stavins, R. (2011). The problem of the commons: still unsettled after 100 years. American Economic Review 101, 81-108.

Ulph, A. and D. Ulph (1997). Global warming, irreversibility and learning. Economic Journal 10\%, 636-650.

White, C. and C. Costello (2014). Close the high seas to fishing? PLoS biology 12(3), e1001826. 